\title{
Electrical conductivity measurements of association phenomena for arginine complexes with some transition metals in mono and disaccharide _ water mixture at $310.16 \mathrm{~K}$
}

\author{
Hala A. A. Chilmeran \\ Department of Animal Production Technique \\ College of Agricultural Technical \\ Northern Technical University \\ dr_hala.awf.chilmeran@ntu.edu.iq
}

\author{
Yasir O. H. Al-Allaf \\ Department of Chemistry \\ College of Science \\ Mosul University \\ yaser alallaf@yahoo.com
}

DOI: $\underline{10.33899 / \text { edusj.2019.162975 }}$

\section{Received}

24 / 12 / 2018
Accepted

16 / 05 / 2019

\begin{abstract}
In the present study we have measured the electrical conductivity of $\mathrm{Mn}$ (II), $\mathrm{Ni}(\mathrm{II}), \mathrm{Fe}(\mathrm{II})$ complexes with Arginine in aqueous solutions of mono and di saccharide at $(310.16 \mathrm{~K})$, The data were analyzed using Lee-Wheaton(LW) conductivity equation for asymmetrical electrolytes. The conductivity parameter: ionic conductivity $(\lambda)$ association constant $\left(\mathrm{K}_{\mathrm{A}}\right)$ and the average distance parameter between the ions (R) at the best fit values of the standard deviation $(\sigma \Lambda)$ have been calculated, The results are discussed depends on the basis of solvent effect on the conductivity parameter of these complexes. Walden products have also been calculated for each solvent composition.
\end{abstract}

Key words: Lee-Wheaton, Arginine, ionic conductivity, Walden product 


\section{قياسات التوصيلية الكهربائية لظاهرة التجمع الايوني لمعقدات الحامض الاميني الارجنين مع عدد من العناصر الانتقالية في محاليل السكريات الاحادية والثنائية عند 310.16مطلقة الائية}

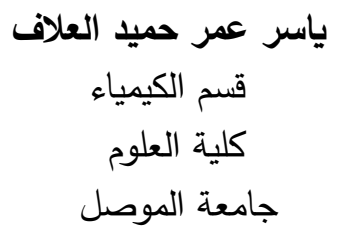

yaser alallaf@yahoo.com
حلا عوف عبد الرحمن جلميران

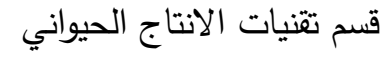

الكلية التقنية الزراعية

الجامعة التقنية الثمالية

dr hala.awf.chilmeran@ntu.edu.iq

DOI: $\underline{10.33899 / \text { edusj.2019.162975 }}$

القبول 2019 / 05 / 16

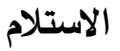

2018 / 12 / 24

\section{الخلاصة}

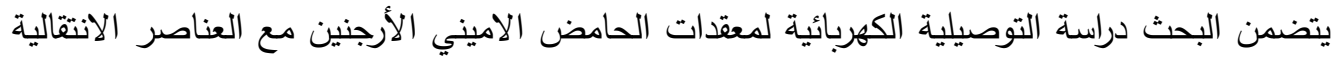
في المحاليل المائية للسكريات الأحادية والثنائية عند درجة حرارة (II) , Ni(II), Fe(II)

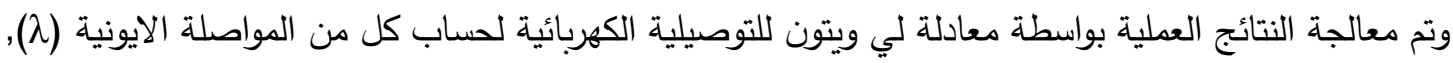

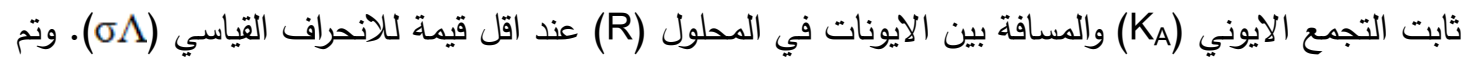

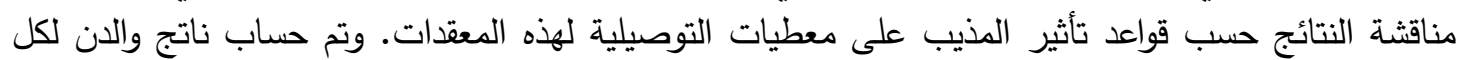
تركيب من المذيب.

الكلمات المفتاحية: التوصيلية الأيونية، الأرجنين، لي ويتون، ناتج والدن.

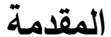

نظرا لأهمية معقدات العناصر الانتقالية مع الاحماض الامينية من الناحية البايولوجية إذ إستعملت الإنة

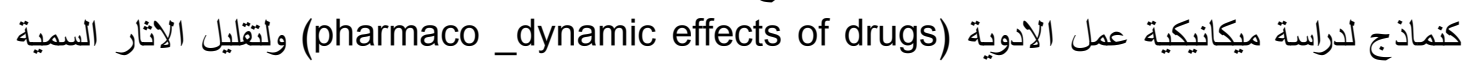

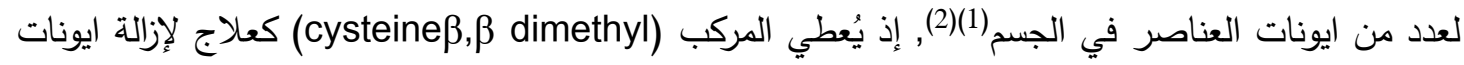

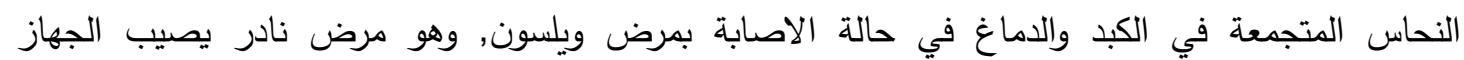

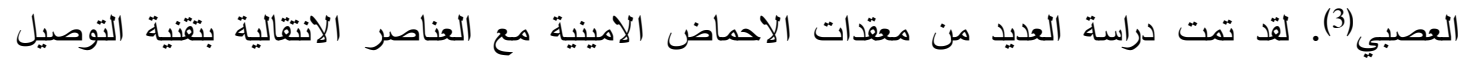

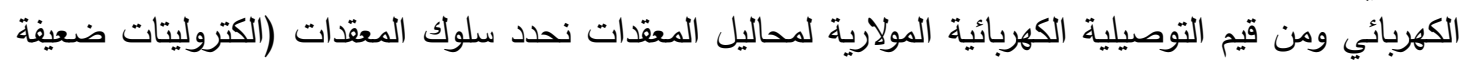

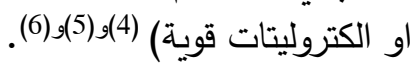

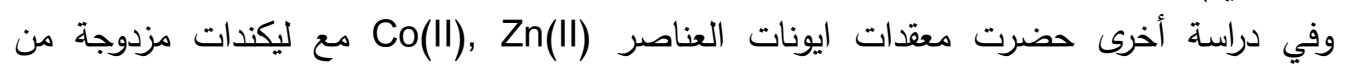

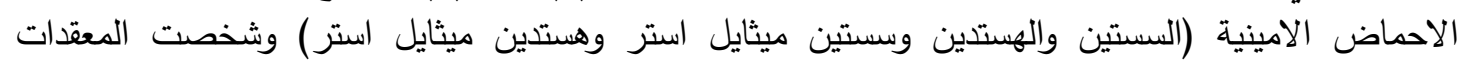

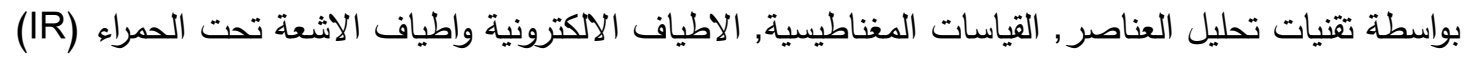

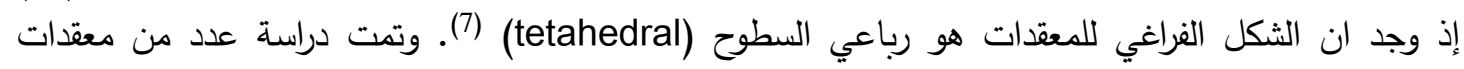

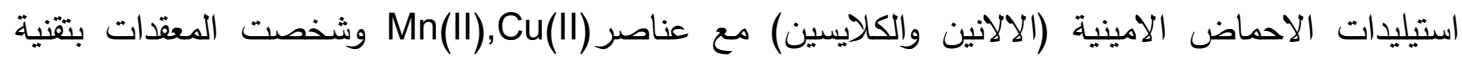
تحليل العناصر والاطياف الالكترونية واطياف الاشعة تحت الحمراء (IR), ومن نتائج القياسات وجد الإندان ان الثكل

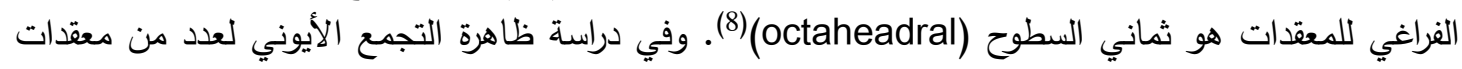

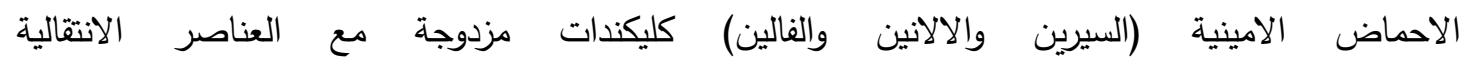
وبنسبة مولية (1:3:3 M(II),Ni(II),Co(II),Ca(II) 


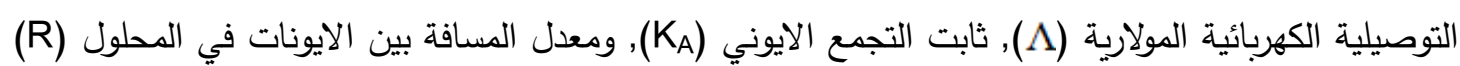

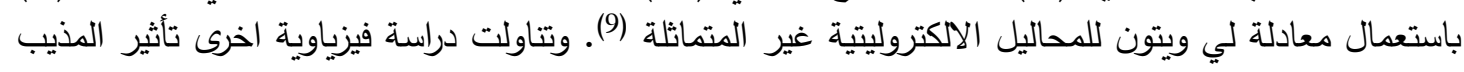

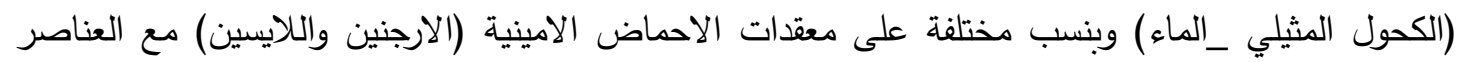

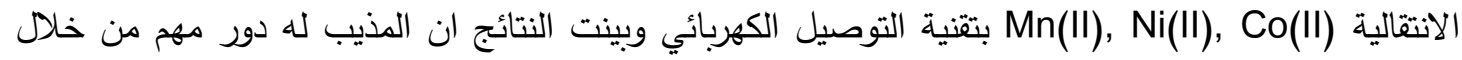

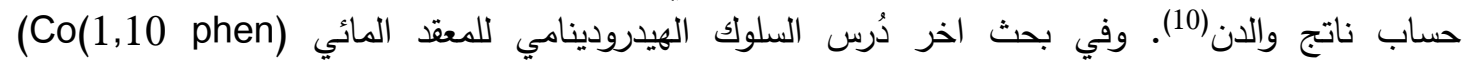
وبنسبة (1:1) في مزيج من الماء والميثانول كمذيب بواسطة قياسات التوصيلية في درجات حرارية مختلفة ووجد ان القوة الكولومية تؤدي دوبة دوراء مها في عملية التجمع الايوني من وجهة نظر

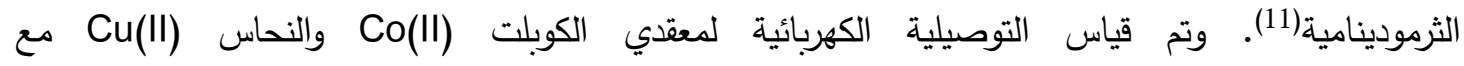

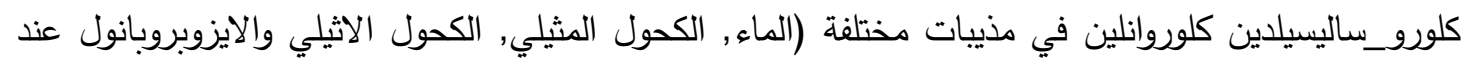

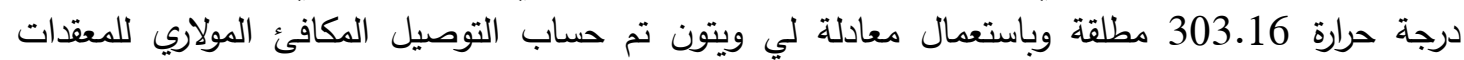

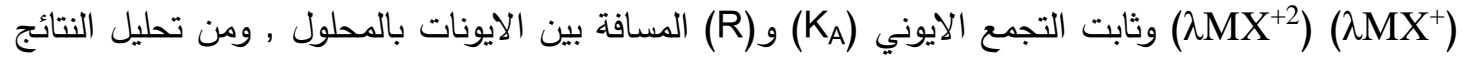

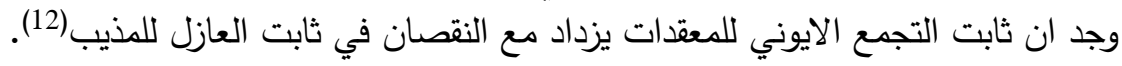

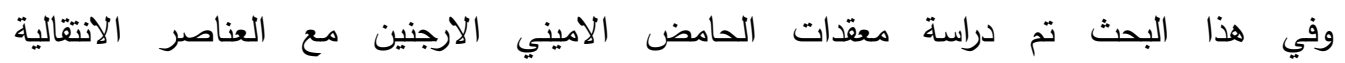

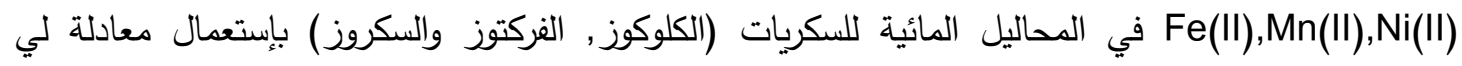

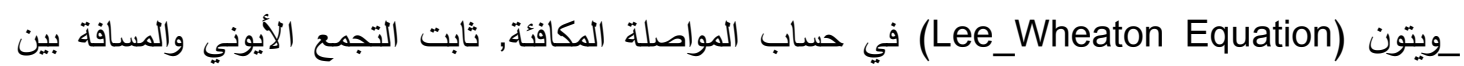
الأيونات في المحلول وتعد هذه المعادلة الصيغة المحدة لمعادلة ديباي_هيكل لحساب المواصلة المكافئة

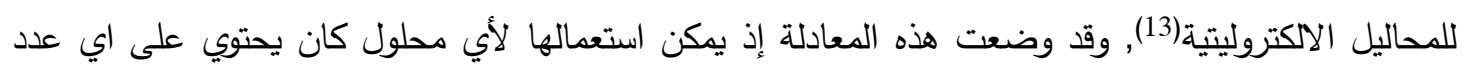
من الايونات وعلى اي نوع من التكافؤ وبالتالي يمكن استعمالها مع الالكتروليتات المتماثلة وغير المتماثلة,

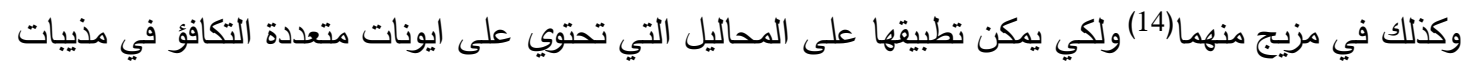

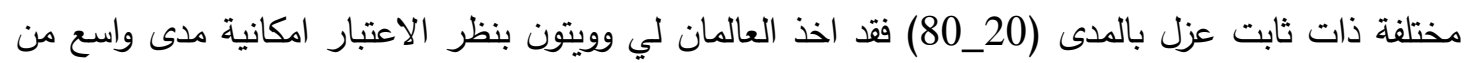

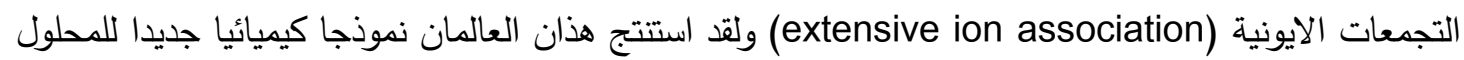

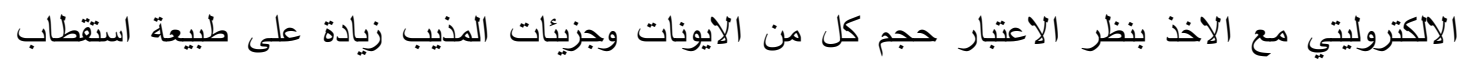
المذيب. وتضمن هذا النموذج مفهوم التجمع الايوني وتكوين الازواج الايونية.

\section{الجزء العملي}

المواد الكيميائية المستعملة جميعها كانت على درجة عالية من النقاوة وهي من انتاج شركتي Fluka و BDH تم تحضير ماء قياس التوصيلية (Deionized Water) وذلك بإعادة تقطير الماء ثناث مرات بعد إضـافة (1.5) غرام من مسحوق برمنكنات البوتاسيوم (KMnO4) الى (1500) مليليتر من الماء المقطر مـع

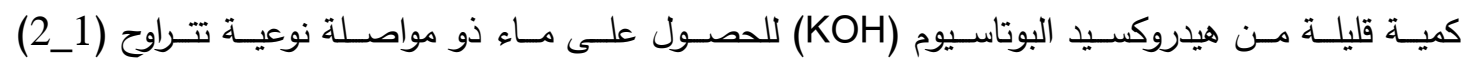

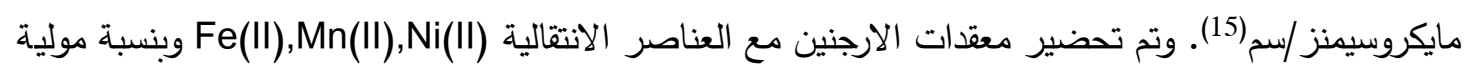
(1:3) مـن مnCl

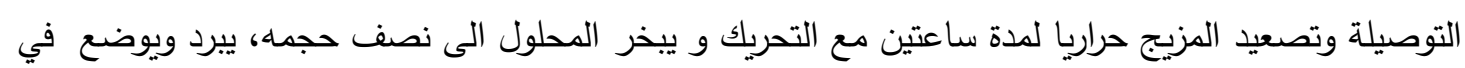

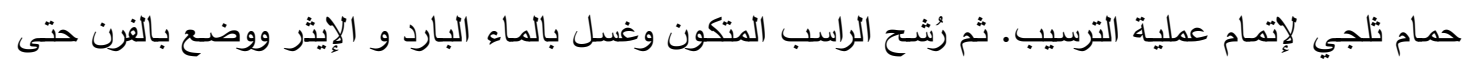
يجف(16). تم إجراء عدد من القياسات الفيزيائية ليتسنى تثخيص الإمام المعقد الناتج. 
القياسات الفيزيائية للمعقدات:

قياسات الاطياف الاككترونية:

Shimadzu, UV_1650 (أجريت قياسات الاطياف الاككترونية للمعقدات المحضرة باستعمال جهاز

وتم القياس عند درجة حرارة الغرفة, إذ استعملت PC_Specctro photometer,UV_Vis Recording) خلايا الكوارتز بعرض (1سم) وضمن المدى (190_1100) نانوميتر .

قياسات أطياف الاشعة تحت الحمراء :

اجريت قياسات اطياف الاشعة تحت الحمراء للمعقدات المحضرة فضلا عن الليكندات وذلك باستعمال

جهاز من نوع) (Tensor 27 CO.Brucker (FT.IR)spectrophotometer) في المنطقة المحصورة مابين (400_4000) سم -1. والجدول (1-1) يوضح نتائج قياسات الأشعة تحت الحمراء.

الجدول(1-1): يبين حزم امتصاص الاشعة تحت الحمراء والاشعة فوق البنفجية - المرئية لليكاند الأرجنين ومعقداته المحضرة

\begin{tabular}{|c|c|c|c|c|c|c|c|c|}
\hline \multirow{2}{*}{ المركب } & \multicolumn{6}{|c|}{ I.R (KBr) band $\mathrm{cm}^{-1}$} & \multirow{2}{*}{$\begin{array}{l}\text { U.V-Vis. } \\
\lambda_{\max } \mathrm{nm}\end{array}$} & \multirow{2}{*}{$\begin{array}{c}\text { U.V-Vis. } \\
\lambda_{\max } \mathrm{cm}^{-1}\end{array}$} \\
\hline & N-H & O-H & $\mathrm{C}-\mathrm{N}$ & $\mathrm{C}=\mathrm{O}$ & M-N & M-O & & \\
\hline Arginine & 2951 & 2865 & 1377 & 1641 & --- & --- & 268 & 37313.432 \\
\hline$\left[\mathrm{Fe}(\operatorname{Arg})_{3}\right] \mathrm{Cl}_{2}$ & 3296 & 3126 & 1329 & 1672 & 448 & 550 & 255 & 28169.014 \\
\hline$\left[\mathrm{Mn}(\operatorname{Arg})_{3}\right] \mathrm{Cl}_{2}$ & 3329 & 3131 & 1358 & 1667 & 427 & 540 & 280 & 35714.285 \\
\hline$\left[\mathrm{Ni}(\mathrm{Arg})_{3}\right] \mathrm{Cl}_{2}$ & 3256 & 3147 & 1361 & 1682 & 409 & 528 & 257 & 38910.505 \\
\hline
\end{tabular}

قياسات التوصيلية لمحاليل المعقدات المحضرة :

تم ضبط درجة حرارة الحمام المائي (Thermal Water bath) .(LAUDA-THERMOSTAT, type S15/12, Made in Western Germany) بدرجة (310.16) مطلقة ثم أخذ (50) مليليتر من المذيب في خلية القياس وترك لحين وصول درجة حرارته

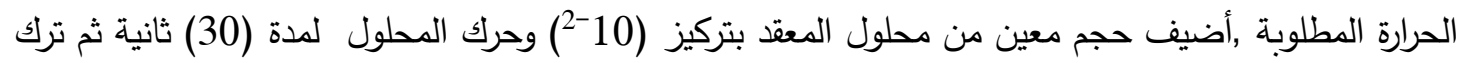

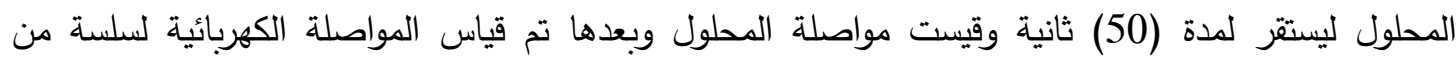
الإضافات المتعاقبة وبالطريقة نفسها تم قياس المواصلة الكهربائية لجميع محاليل المعقدات المحضرة ولئه

\section{قياسات اللزوجة للمحاليل السكربة:}

بعد تحضير المحاليل السكرية وبالنسب المئوية المذكورة سابقاً، تم قياس اللزوجة لكل محلول عند درجة

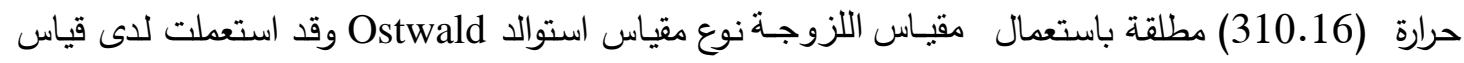
اللزوجة انابيب شعرية مختلفة (K=0.1, 1) إذ أن K هي (نصف قطر الانبوبة الثعرية لمقياس اللزوجة) وذلك بسبب الاختلاف في الكثافة للمحاليل السكرية, وقد أجريت القياسات باستعمال حمام مائي منظم حرارياً والجدول ادناه يوضح قيم اللزوجة للمحاليل السكرية وبدرجة (310.16) مطلقة. 
قياسات التوصيلية الكهربائية لظاهرة التجمع الايوني لمعقدات الحامض الاميني الارجنين مع عدد من العناصر ...

جدول (1-2): يوضح قيم اللزوجة بوحدة (سنتي بوازCp) لمحاليل السكريات في درجة حرارة (310,16) مطلقة

\begin{tabular}{|c|c|c|c|}
\hline Wt\% & Glucose sugar & Fructose sugar & Sucrose sugar \\
\hline \hline $5 \%$ & 0.7084 & 0.6940 & 0.7289 \\
\hline $10 \%$ & 0.7429 & 0.7331 & 0.7736 \\
\hline $15 \%$ & 0.7674 & 0.7519 & 0.8291 \\
\hline $20 \%$ & 0.8019 & 0.7846 & 0.8431 \\
\hline
\end{tabular}

الجدول (1-3): يبين الصيغ الوضعية للحامض الأميني الأرجنين ومعقداته المحضرة مع بعض الصفات الفيزيائية

\begin{tabular}{|c|c|c|c|}
\hline رقم المقدد & الصيغة الوضعية للمعقد & 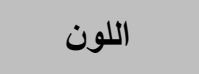 & درجة الإنصهار (م) \\
\hline 1 & Arginine & أبيض & $235-238$ \\
\hline 2 & {$\left[\mathrm{Fe}(\mathrm{Arg})_{3}\right] \mathrm{Cl}_{2}$} & بني ( محمر ) & $167-170$ \\
\hline 3 & {$\left[\mathrm{Mn}(\mathrm{Arg})_{3}\right] \mathrm{Cl}_{2}$} & ترابي & $173-175$ \\
\hline 4 & {$\left[\mathrm{Ni}(\operatorname{Arg})_{3}\right] \mathrm{Cl}_{2}$} & 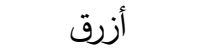 & $118-120$ \\
\hline
\end{tabular}

\section{النتائج والمناقشة}

Fe(II),Mn(II),Ni(II)(تم دراسة المواصلة الكهربائية لمحاليل معقدات الأرجنين مع العناصر الانتقالئهائل

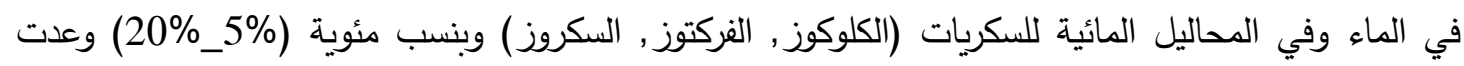

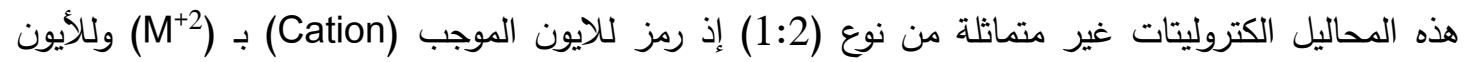

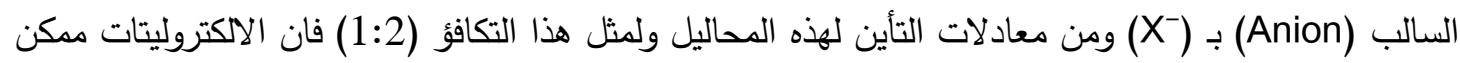
$\mathrm{M}^{+2}+\mathrm{X}^{-} \mathrm{K}^{+}$ ان تتأين على النحو الاتي: $\mathrm{MX}^{+}+\mathrm{X}^{-} \stackrel{\mathrm{K}_{\mathrm{A}}(2)}{=} \mathrm{MX}_{2}$

(association constant) ثابت التجمع الايوني K $\left[\mathrm{M}(\mathrm{Arg})_{3}\right]^{+2}: \mathrm{M}^{+2}$

$\mathrm{Cl} \quad: \mathrm{X}^{-}$

[ M(Arg) $\left.{ }_{3} \mathrm{Cl}\right]^{-}$الايون الوسطي : MXX

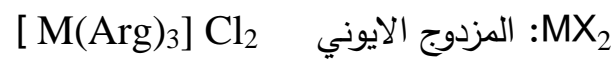
الاملاح المستخدمة : اذن يوجد ثلاثة انواع من الايونات وهي MX+ , X' , M+2 يحصل تجمع ايوني في الاملاح من نوع (1:2) ويعبر عنها كما يلي: $\Lambda \mathrm{MX}_{2}=\mathrm{f}\left(\lambda^{\circ} \mathrm{M}^{+2}, 2 \lambda^{\circ} \mathrm{MX}^{+}, \lambda^{\circ} \mathrm{X}^{-}, \mathrm{KA}(1), \mathrm{KA}(2), \mathrm{R}\right)$

حيث ان (R) هي معدل المسافة بين الايونين.

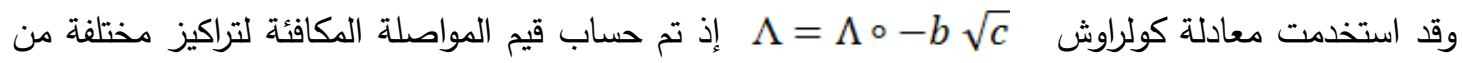

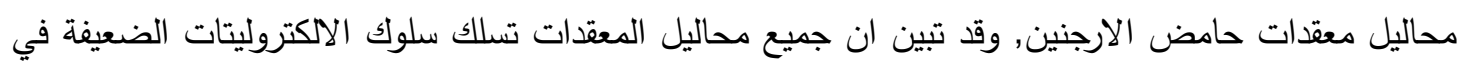


الماء وفي المحاليل المائية للسكريات (الكلوكوز , الفركتوز , السكروز) وتم إثبات ذلك من خلال رسم العلاقة بين

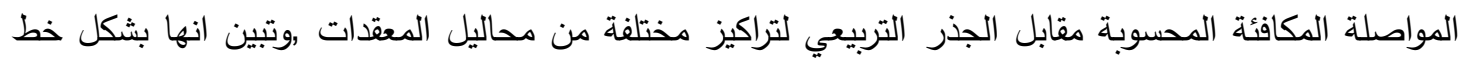

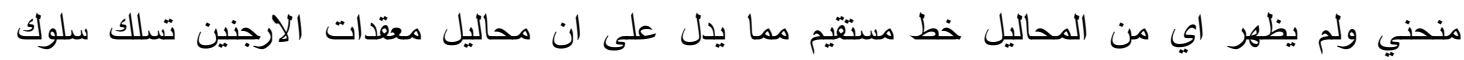
الاكتروليتات الضعيفة في الماء وفي المحاليل المائية للسكريات الاحادية والثنائية وكما موضح بالئ بالأشكال أدناه ومن الجداول (1-3) إلى (1-12) نلاحظ ان السلوك العام لمحاليل معقدات الارجنين مع العناصر الانتقالية انها الكتروليتات ضعيفة.

جلول (1-3): يوضح قيم الجذر التربيعي لتراكيز مختلفة لمعقدات الحامض الأميني الأرجنين إزاء المواصلة المكافئة

في الماء وعند درجة حرارة (310.16 مطلقة)

\begin{tabular}{|c|c|c|c|c|c|}
\hline \multicolumn{2}{|c|}{$\left[\mathrm{Fe}(\mathrm{Arg})_{3}\right] \mathrm{Cl}_{2}$} & \multicolumn{2}{c|}{$\left[\mathrm{Mn}(\mathrm{Arg})_{3}\right] \mathrm{Cl}_{2}$} & \multicolumn{2}{c|}{$\left[\mathrm{Ni}(\mathrm{Arg})_{3}\right] \mathrm{Cl}_{2}$} \\
\hline$\sqrt{\text { conc. } \times 10^{2}}$ & $\Delta$ equiv. & $\sqrt{\text { conc. } \times 10^{2}}$ & $\Delta$ equiv. & $\sqrt{\text { conc. } \times 10^{2}}$ & $\Delta$ equiv. \\
\hline 1.3985 & 48.2434 & 1.4054 & 42.8016 & 1.4380 & 43.7543 \\
\hline 1.9593 & 42.6836 & 1.9671 & 41.9969 & 2.0079 & 41.7674 \\
\hline 2.3769 & 40.0664 & 2.3827 & 41.8616 & 2.4336 & 39.1554 \\
\hline 2.7174 & 38.5761 & 2.7230 & 41.6353 & 2.7790 & 38.6172 \\
\hline 3.0087 & 38.5415 & 3.0143 & 41.4394 & 3.0728 & 38.5279 \\
\hline 3.2625 & 38.3167 & 3.2664 & 41.2390 & 3.3286 & 38.3818 \\
\hline 3.4908 & 38.0362 & 3.4944 & 41.0453 & 3.5594 & 38.2308 \\
\hline 3.6960 & 37.8334 & 3.7010 & 40.9341 & 3.7676 & 38.2150 \\
\hline 3.8832 & 37.8253 & 3.8884 & 40.8978 & 3.9587 & 38.0758 \\
\hline 4.0557 & 37.5057 & 4.0601 & 40.7244 & 4.1321 & 38.0043 \\
\hline 4.2151 & 37.2380 & 4.2201 & 40.5438 & 4.2925 & 37.8223 \\
\hline 4.3640 & 37.2278 & 4.3681 & 40.4641 & 4.4393 & 37.0186 \\
\hline 4.5021 & 37.0806 & 4.5068 & 40.3918 & 4.5798 & 36.8977 \\
\hline 4.6314 & 37.0303 & 4.6352 & 40.3156 & 4.7098 & 36.2288 \\
\hline 4.7548 & 37.0288 & 4.7591 & 40.2697 & 4.8327 & 35.9830 \\
\hline
\end{tabular}

شكل (1-1): العلاقة بين الجذر التربيعي لتراكيز معقدات حامض الأرجنين إزاء المواصلة المكافئة في الماء بدرجة

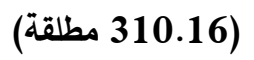

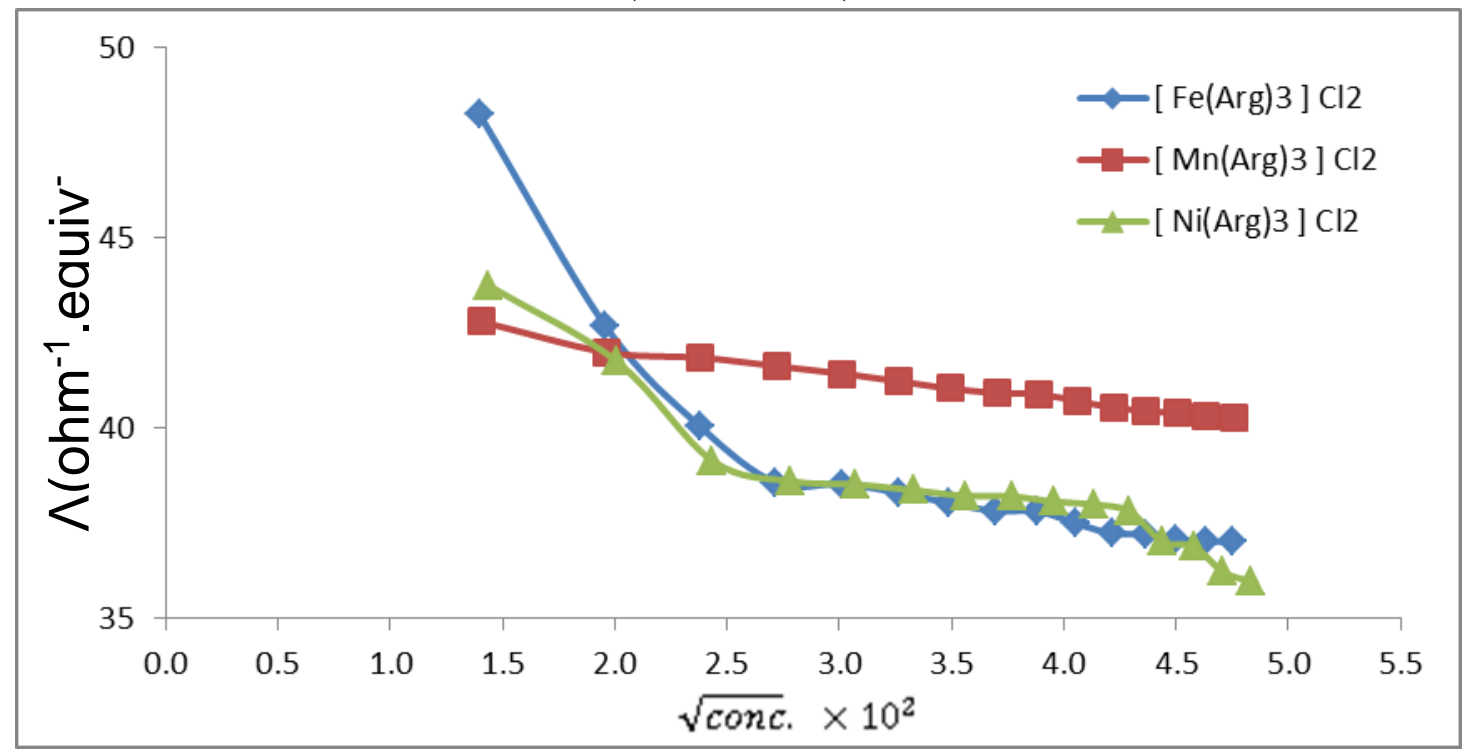


جدول (1-4): يوضح قيم الجذر التربيعي لتراكيز مختلفة لمعقدات الحامض الأميني الأرجنين مع الحديد إزاء المواصلة المكافئة بنسب مختلفة من محلول الكلوكوز عند درجة حرارة (310.16 مطلقة)

\begin{tabular}{|c|c|c|c|c|c|c|c|}
\hline \multicolumn{2}{|c|}{$5 \%$ Glucose } & \multicolumn{2}{c|}{$10 \%$ Glucose } & \multicolumn{2}{c|}{$15 \%$ Glucose } & \multicolumn{2}{c|}{$20 \%$ Glucose } \\
\hline$\sqrt{\text { conc. } \times 10^{2}}$ & $\Lambda$ equiv. & $\sqrt{\text { conc. } \times 10^{2}}$ & $\Delta$ equiv. & $\sqrt{\text { conc. } \times 10^{2}}$ & $\Lambda$ equiv. & \multicolumn{1}{c|}{ conc. $\times 10^{2}$} & $\Delta$ equiv. \\
\hline 1.3950 & 55.5542 & 1.3918 & 52.1894 & 1.3951 & 45.1001 & 1.3918 & 38.8802 \\
\hline 1.9503 & 45.6974 & 1.9471 & 44.2581 & 1.9529 & 39.6664 & 1.9471 & 35.6932 \\
\hline 2.3597 & 43.2493 & 2.3585 & 40.8612 & 2.3655 & 36.8651 & 2.3585 & 34.1249 \\
\hline 2.6962 & 41.2005 & 2.6916 & 39.7915 & 2.7013 & 35.9733 & 2.6916 & 32.8607 \\
\hline 2.9839 & 40.9691 & 2.9774 & 39.7396 & 2.9885 & 35.8461 & 2.9774 & 32.6946 \\
\hline 3.2375 & 40.8642 & 3.2323 & 39.5101 & 3.2421 & 35.6008 & 3.2323 & 32.6872 \\
\hline 3.4628 & 40.3909 & 3.4574 & 38.0462 & 3.4672 & 35.1518 & 3.4574 & 32.6199 \\
\hline 3.6669 & 39.9236 & 3.6594 & 37.9567 & 3.6693 & 35.1275 & 3.6594 & 32.4827 \\
\hline 3.8543 & 39.0983 & 3.8454 & 37.6542 & 3.8540 & 34.8674 & 3.8454 & 32.4109 \\
\hline 4.0247 & 38.5422 & 4.0159 & 37.3137 & 4.0248 & 34.7798 & 4.0159 & 32.3543 \\
\hline 4.1827 & 37.8005 & 4.1735 & 37.1700 & 4.1828 & 34.6816 & 4.1735 & 32.3242 \\
\hline 4.3311 & 37.3337 & 4.3214 & 37.1408 & 4.3298 & 34.5232 & 4.3214 & 32.3040 \\
\hline 4.4674 & 37.1708 & 4.4573 & 37.1050 & 4.4661 & 34.4201 & 4.4573 & 32.2463 \\
\hline 4.5935 & 37.0057 & 4.5832 & 37.0841 & 4.5937 & 34.3706 & 4.5832 & 32.2267 \\
\hline 4.7129 & 36.9769 & 4.7024 & 36.9633 & 4.7144 & 34.3662 & 4.7024 & 32.1456 \\
\hline
\end{tabular}

شكل (1-2): العلاقة بين الجذر التربيعي لتراكيز معقدات حامض الأرجنين مع الحديد إزاء المواصلة المكافئة بنسب مختلفة في محلول الكلوكوز بلرجة (310.16 مطلقة)

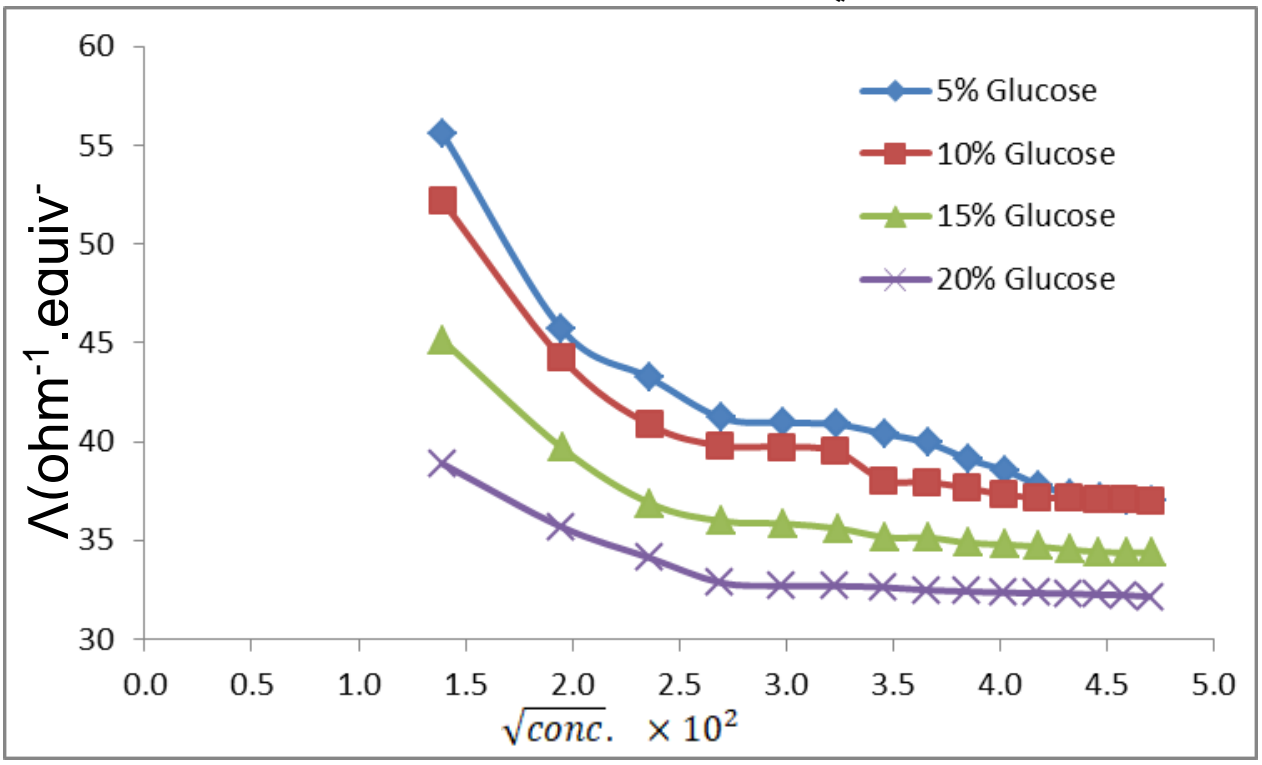


جدول (1-5): يوضح قيم الجذر التربيعي لتراكيز مختلفة لمققدات الحامض الأميني الأرجنين مع الحديد إزاء المواصلة المكافئة بنسب مختلفة من محلول الفركتوز عند درجة حرارة (310.16 مطلقة)

\begin{tabular}{|c|c|c|c|c|c|c|c|}
\hline \multicolumn{2}{|c|}{$5 \%$ Fructose } & \multicolumn{2}{c|}{$10 \%$ Fructose } & \multicolumn{2}{c|}{$15 \%$ Fructose } & \multicolumn{2}{c|}{$20 \%$ Fructose } \\
$\sqrt{\text { conc. } \times 10^{2}}$ & $\Lambda$ equiv. & conc. $\times 10^{2}$ & $\Lambda$ equiv. & $\sqrt{\text { conc. } \times 10^{2}}$ & $\Lambda$ equiv. & $\sqrt{\text { conc. } \times 10^{2}}$ & $\Delta$ equiv. \\
\hline 1.4053 & 79.0452 & 1.4049 & 71.3529 & 1.4049 & 62.3881 & 1.3988 & 61.4930 \\
\hline 1.9680 & 53.8128 & 1.9591 & 50.2473 & 1.9591 & 49.7280 & 1.9538 & 45.1423 \\
\hline 2.3855 & 44.2063 & 2.3702 & 45.8113 & 2.3702 & 44.2675 & 2.3681 & 39.8232 \\
\hline 2.7279 & 43.2790 & 2.7087 & 44.2088 & 2.7087 & 40.8455 & 2.7373 & 35.8762 \\
\hline 3.0177 & 42.3924 & 2.9937 & 42.2736 & 2.9937 & 40.4679 & 3.0225 & 35.8282 \\
\hline 3.2730 & 41.2640 & 3.2495 & 41.0399 & 3.2496 & 39.0807 & 3.2689 & 34.5612 \\
\hline 3.4996 & 40.5442 & 3.4753 & 39.9802 & 3.4754 & 37.9769 & 3.4898 & 33.7728 \\
\hline 3.7046 & 40.0077 & 3.6829 & 39.1381 & 3.6830 & 37.7261 & 3.6898 & 33.3334 \\
\hline 3.8873 & 39.8565 & 3.8687 & 38.8694 & 3.8688 & 37.1524 & 3.8776 & 32.7701 \\
\hline 4.0532 & 39.7602 & 4.0425 & 38.8058 & 4.0422 & 37.0221 & 4.0504 & 32.7437 \\
\hline 4.2088 & 39.7418 & 4.2008 & 38.3765 & 4.1979 & 36.8973 & 4.2065 & 32.6274 \\
\hline 4.3524 & 39.4045 & 4.3480 & 38.3629 & 4.3445 & 36.5408 & 4.3520 & 32.5758 \\
\hline 4.4900 & 39.3306 & 4.4841 & 38.3429 & 4.4820 & 36.3581 & 4.4886 & 32.4609 \\
\hline 4.6186 & 39.2941 & 4.6118 & 38.2986 & 4.6118 & 36.3569 & 4.6164 & 32.3251 \\
\hline 4.7415 & 39.0109 & 4.7334 & 38.2059 & 4.7346 & 35.7846 & 4.7385 & 32.2590 \\
\hline
\end{tabular}

شكل (1-3): العلاقة بين الجذر التربيعي لتراكيز معقدات حامض الأرجنين مع الحديد إزاء المواصلة المكافئة بنسب

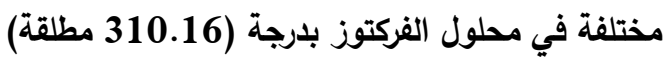

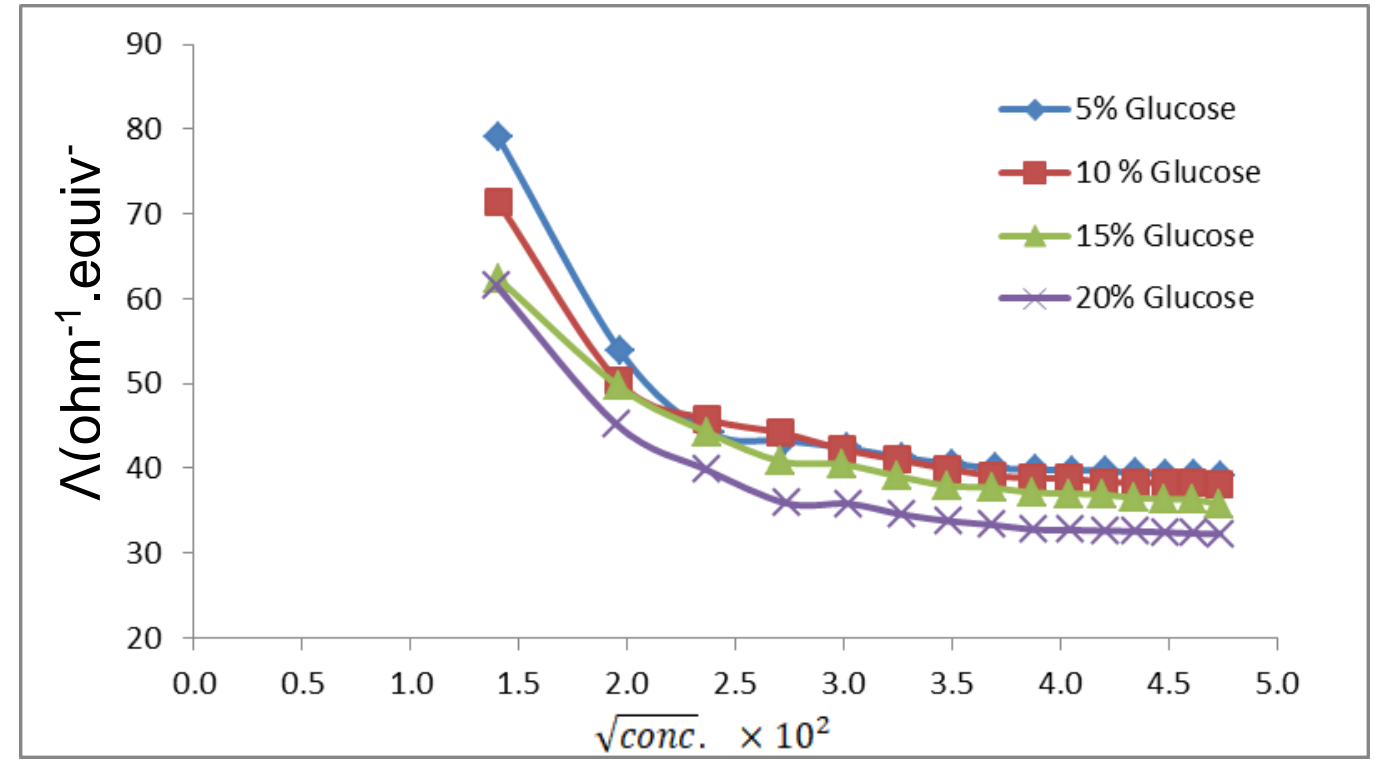


جدول (1-6): يوضح قيم الجذر التربيعي لتراكيز مختلفة لمققدات الحامض الأميني الأرجنين مع الحديد إزاء المواصلة المكافئة بنسب مختلفة من محلول السكروز عند درجة حرارة (310.16 مطلقة)

\begin{tabular}{|c|c|c|c|c|c|c|c|}
\hline \multicolumn{2}{|c|}{$5 \%$ Sucrose } & \multicolumn{2}{c|}{$10 \%$ Sucrose } & \multicolumn{2}{c|}{$15 \%$ Sucrose } & \multicolumn{2}{c|}{$20 \%$ Sucrose } \\
\hline$\sqrt{\text { conc. } \times 10^{2}}$ & $\Lambda$ equiv. & $\sqrt{\text { conc. } \times 10^{2}}$ & $\Delta$ equiv. & $\sqrt{\text { conc. } \times 10^{2}}$ & $\Lambda$ equiv. & $\sqrt{\text { conc. } \times 10^{2}}$ & $\Lambda$ equiv. \\
\hline 1.3963 & 54.2353 & 1.4007 & 56.9296 & 1.3963 & 58.9852 & 1.4180 & 60.8823 \\
\hline 1.9535 & 46.8404 & 1.9595 & 43.8563 & 1.9534 & 44.1712 & 1.9823 & 42.9816 \\
\hline 2.3657 & 43.2847 & 2.3729 & 41.1311 & 2.3619 & 39.5355 & 2.3947 & 37.9959 \\
\hline 2.7050 & 41.6666 & 2.7130 & 39.0721 & 2.7049 & 37.0472 & 2.7340 & 36.4415 \\
\hline 2.9950 & 41.3081 & 3.0037 & 38.9150 & 2.9922 & 36.4743 & 3.0263 & 34.7113 \\
\hline 3.2455 & 40.8474 & 3.2547 & 38.1007 & 3.2453 & 35.7526 & 3.2781 & 33.7707 \\
\hline 3.4715 & 40.3075 & 3.4811 & 37.3485 & 3.4691 & 35.5265 & 3.5053 & 33.0748 \\
\hline 3.6735 & 40.0702 & 3.6836 & 37.2626 & 3.6734 & 34.6127 & 3.7079 & 32.3607 \\
\hline 3.8590 & 39.5350 & 3.8694 & 36.8841 & 3.8570 & 34.2196 & 3.8952 & 31.9259 \\
\hline 4.0300 & 39.3846 & 4.0407 & 36.7444 & 4.0298 & 33.6872 & 4.0657 & 31.4217 \\
\hline 4.1894 & 39.1444 & 4.2003 & 36.6401 & 4.1876 & 33.4203 & 4.2244 & 31.2912 \\
\hline 4.3376 & 39.0785 & 4.3487 & 36.3473 & 4.3373 & 33.2260 & 4.3716 & 30.9976 \\
\hline 4.4750 & 39.0366 & 4.4864 & 36.2022 & 4.4734 & 33.0597 & 4.5068 & 30.9090 \\
\hline 4.6036 & 39.0211 & 4.6151 & 36.0541 & 4.6033 & 32.8247 & 4.6346 & 30.8887 \\
\hline 4.7252 & 38.9631 & 4.7369 & 35.9621 & 4.7236 & 32.7642 & 4.7552 & 30.8639 \\
\hline
\end{tabular}

شكل (1-4): العلاقة بين الجذر التربيعي لتراكيز معقدات حامض الأرجنين مع الحديد إزاء المواصلة المكافئة بنسب مختلفة في محلول السكروز بارجة (310.16 مطلقة)

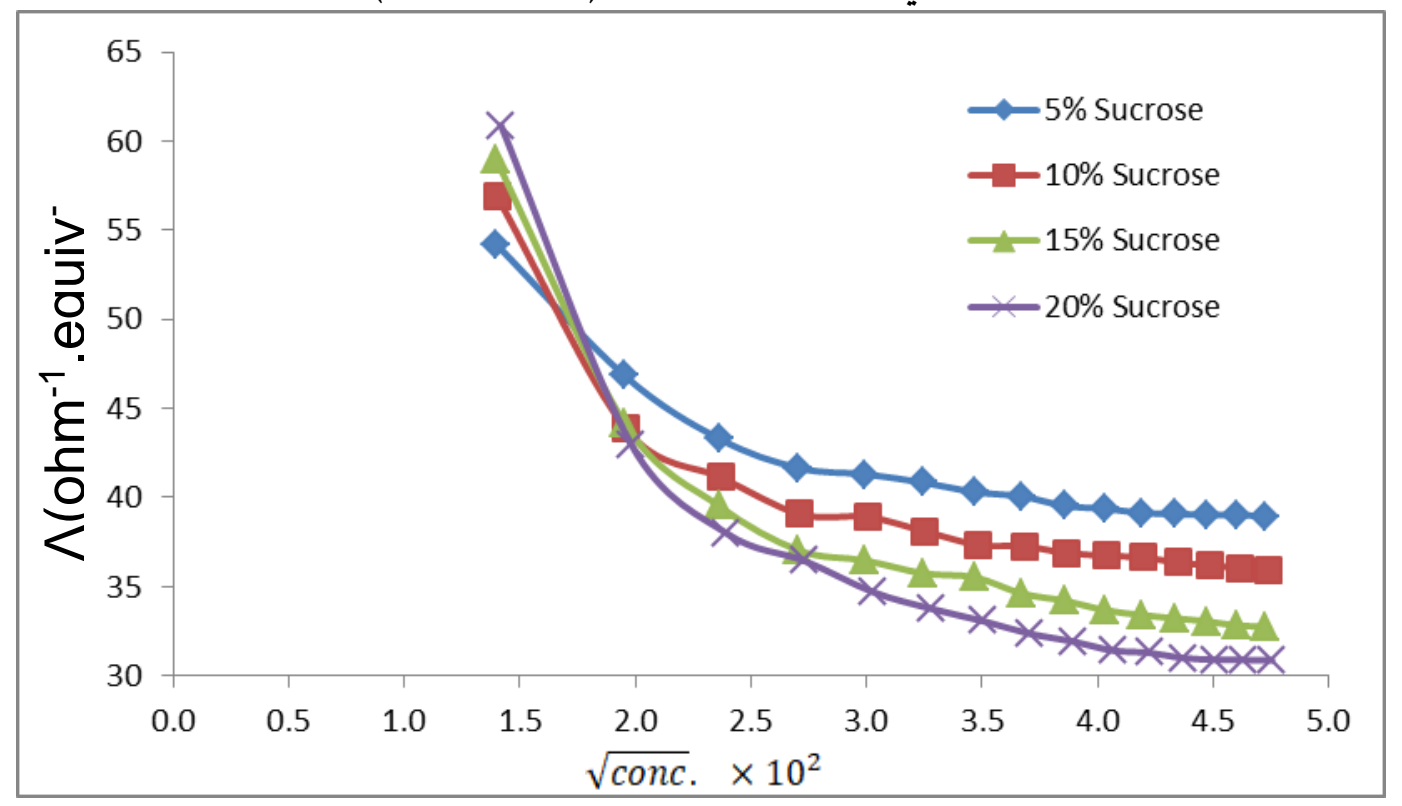


جدول (1-7): يوضح قيم الجذر التربيعي لتراكيز مختلفة لمعقدات الحامض الأميني الأرجنين مع المنغنيز إزاء المواصلة المكافئة بنسب مختلفة من محلول الكلوكوز عند درجة حرارة (310.16 مطلقة)

\begin{tabular}{|c|c|c|c|c|c|c|c|}
\hline \multicolumn{2}{|c|}{$5 \%$ Glucose } & \multicolumn{2}{|c|}{$10 \%$ Glucose } & \multicolumn{2}{|c|}{$15 \%$ Glucose } & \multicolumn{2}{|c|}{$20 \%$ Glucose } \\
\hline$\sqrt{\text { conc. } \times 10^{2}}$ & $\Lambda$ equiv. & $\sqrt{\text { conc. } \times 10^{2}}$ & $\Delta$ equiv. & $\sqrt{\text { conc. } \times 10^{2}}$ & $\Lambda$ equiv. & $\sqrt{\text { conc. } \times 10}$ & $\Delta$ equiv. \\
\hline 1.3955 & 60.8293 & 1.3897 & 58.9523 & 1.3954 & 56.4690 & 1.3959 & 57.1865 \\
\hline 1.9542 & 49.5368 & 1.9482 & 48.3472 & 1.9542 & 44.1108 & 1.9539 & 43.0727 \\
\hline 2.3672 & 45.7811 & 2.3616 & 42.6760 & 2.3671 & 39.7013 & 2.3671 & 37.7339 \\
\hline 2.7021 & 42.6280 & 2.6979 & 40.5306 & 2.7020 & 37.6604 & 2.7017 & 35.8183 \\
\hline 2.9883 & 42.3243 & 2.9832 & 40.5095 & 2.9882 & 37.2329 & 2.9883 & 34.1820 \\
\hline 3.2407 & 41.2980 & 3.2335 & 39.3589 & 3.2407 & 36.0843 & 3.2405 & 33.9230 \\
\hline 3.4657 & 40.7332 & 3.4583 & 38.6726 & 3.4656 & 35.7993 & 3.4656 & 33.4245 \\
\hline 3.6672 & 39.8698 & 3.6611 & 38.0496 & 3.6671 & 35.0213 & 3.6668 & 33.0539 \\
\hline 3.8520 & 39.8010 & 3.8446 & 37.5482 & 3.8520 & 34.8746 & 3.8522 & 32.2609 \\
\hline 4.0218 & 39.5915 & 4.0143 & 37.1415 & 4.0218 & 34.4948 & 4.0218 & 32.1396 \\
\hline 4.1784 & 39.1012 & 4.1705 & 36.5088 & 4.1784 & 34.0770 & 4.1786 & 32.0451 \\
\hline 4.3263 & 39.0263 & 4.3184 & 36.2776 & 4.3263 & 34.0701 & 4.3262 & 31.9032 \\
\hline 4.4629 & 38.6922 & 4.4550 & 36.0520 & 4.4629 & 34.0051 & 4.4629 & 31.8677 \\
\hline 4.5909 & 38.5173 & 4.5830 & 35.9955 & 4.5909 & 33.9462 & 4.5910 & 31.8660 \\
\hline 4.7105 & 38.4619 & 4.7026 & 35.9703 & 4.7105 & 33.9136 & 4.7108 & 31.7826 \\
\hline
\end{tabular}

شكل (1-5): العلاقة بين الجذر التربيعي لتراكيز معقدات حامض الأرجنين مع المنفيز إزاء المواصلة المكافئة بنسب مختلفة في محلول الكلوكوز بدرجة (310.16 مطلقة)

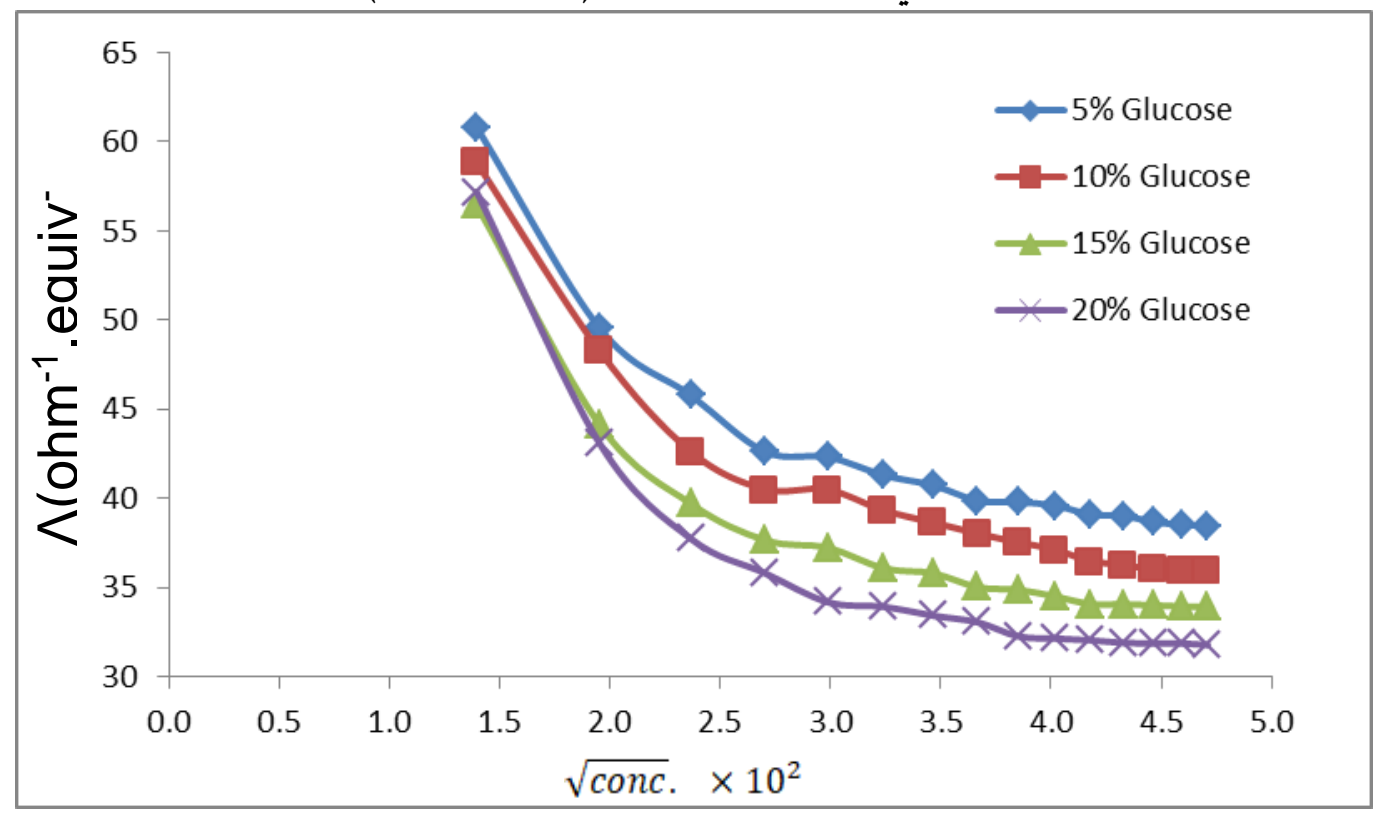


جدول (1-8): يوضح قيم الجذر التربيعي لتراكيز مختلفة لمعقدات الحامض الأميني الأرجنين مع المنفنيز إزاء المواصلة المكافئة بنسب مختلفة من محلول الفركتوز عند درجة حرارة (310.16 مطلقة)

\begin{tabular}{|c|c|c|c|c|c|c|c|}
\hline \multicolumn{2}{|c|}{$5 \%$ Fructose } & \multicolumn{2}{|c|}{$10 \%$ Fructose } & \multicolumn{2}{|c|}{$15 \%$ Fructose } & \multicolumn{2}{|c|}{$20 \%$ Fructose } \\
\hline$\sqrt{\text { conc. } \times 10^{2}}$ & $\Lambda$ equiv. & $\sqrt{\text { conc. } \times 10^{2}}$ & $\Delta$ equiv. & $\sqrt{\text { conc. } \times 10^{2}}$ & $\Delta$ equiv. & $\sqrt{\text { conc. } \times 10^{2}}$ & $\Delta$ equiv. \\
\hline 1.3724 & 63.7724 & 1.3706 & 62.0926 & 1.3739 & 57.7738 & 1.3793 & 59.1202 \\
\hline 1.9339 & 50.9659 & 1.9314 & 49.4648 & 1.9351 & 45.9508 & 1.9343 & 44.8940 \\
\hline 2.3504 & 46.7252 & 2.3473 & 44.2909 & 2.3513 & 41.3411 & 2.3548 & 40.1208 \\
\hline 2.6805 & 46.1541 & 2.6769 & 42.1467 & 2.6820 & 39.2134 & 2.6655 & 38.0692 \\
\hline 2.9677 & 44.5793 & 2.9638 & 41.8392 & 2.9696 & 38.7897 & 2.9605 & 37.5335 \\
\hline 3.2224 & 43.0592 & 3.2182 & 40.5059 & 3.2231 & 38.2695 & 3.2213 & 36.1844 \\
\hline 3.4470 & 42.1332 & 3.4426 & 39.9541 & 3.4477 & 37.2744 & 3.4485 & 35.1466 \\
\hline 3.6518 & 41.5140 & 3.6473 & 39.3555 & 3.6524 & 36.6977 & 3.6615 & 34.4213 \\
\hline 3.8389 & 40.8911 & 3.8342 & 38.7399 & 3.8399 & 36.2206 & 3.8536 & 33.7014 \\
\hline 4.0113 & 40.5724 & 4.0065 & 38.2519 & 4.0126 & 35.7160 & 4.0252 & 33.4505 \\
\hline 4.1733 & 40.4843 & 4.1684 & 37.1807 & 4.1743 & 35.3840 & 4.1849 & 32.9737 \\
\hline 4.3189 & 40.4653 & 4.3140 & 36.8092 & 4.3202 & 35.2971 & 4.3300 & 32.6014 \\
\hline 4.4568 & 40.4510 & 4.4517 & 36.6600 & 4.4582 & 35.1645 & 4.4674 & 32.5335 \\
\hline 4.5884 & 40.3217 & 4.5832 & 36.4429 & 4.5900 & 35.1504 & 4.5961 & 32.3914 \\
\hline 4.7113 & 40.1315 & 4.7062 & 36.3925 & 4.7129 & 35.0977 & 4.7189 & 32.2130 \\
\hline
\end{tabular}

شكل (1-6): العلاقة بين الجذر التربيعي لتراكيز معقدات حامض الأرجنين م المنفنيز إزاء المواصلة المكافئة بنسب

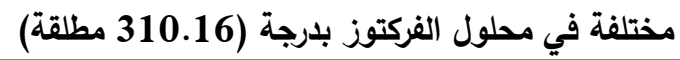

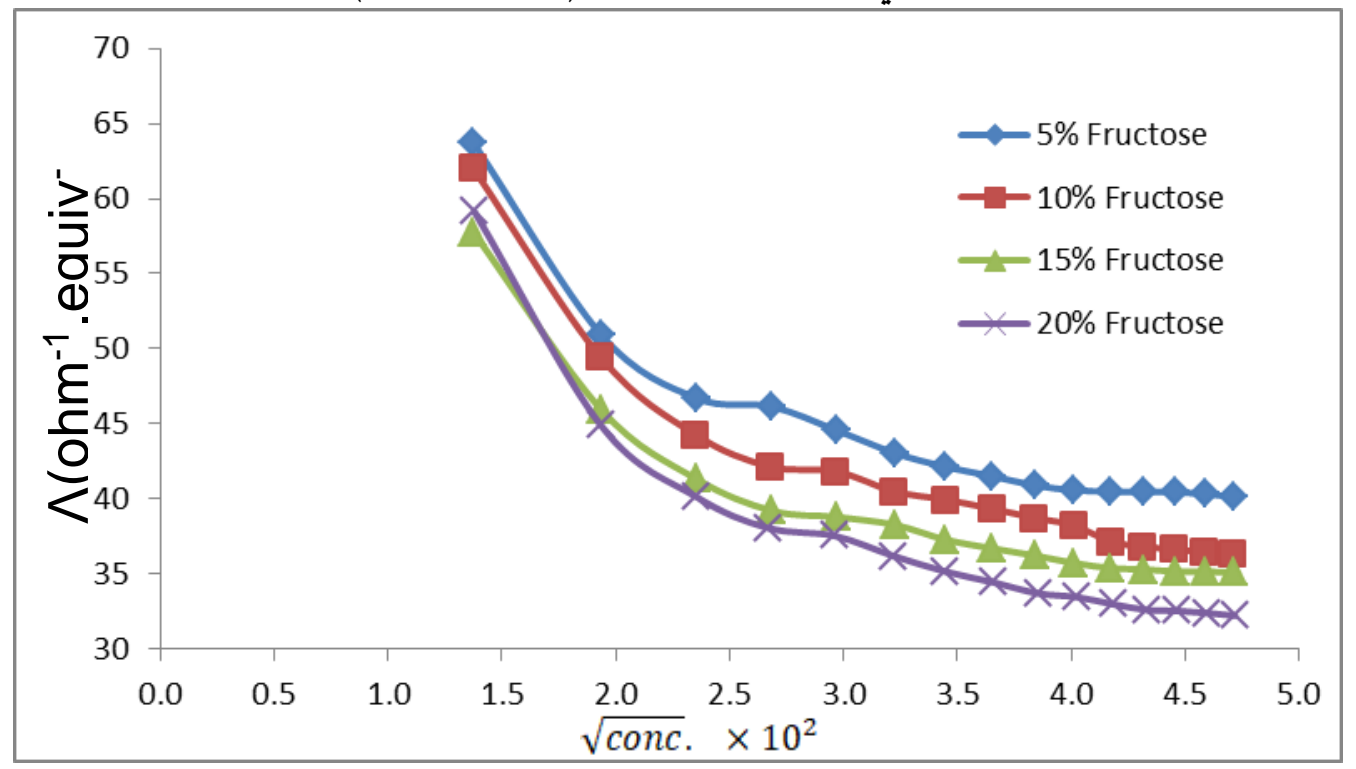


جدول (1-9): يوضح قيم الجذر التربيعي لتراكيز مختلفة لمعقدات الحامض الأميني الأرجنين مع المنغنيز إزاء المواصلة المكافئة بنسب مختلفة من محلول السكروز عند درجة حرارة (310.16 مطلقة)

\begin{tabular}{|c|c|c|c|c|c|c|c|}
\hline \multicolumn{2}{|c|}{$5 \%$ Sucrose } & \multicolumn{2}{c|}{$10 \%$ Sucrose } & \multicolumn{2}{c|}{$15 \%$ Sucrose } & \multicolumn{2}{c|}{$20 \%$ Sucrose } \\
\hline$\sqrt{\text { conc. } \times 10^{2}}$ & A equiv. & conc. $\times 10^{2}$ & $\Delta$ equiv. & $\sqrt{\text { conc. } \times 10^{2}}$ & $\Delta$ equiv. & conc. $\times 10^{2}$ & $\Delta$ equiv. \\
\hline 1.3688 & 48.2191 & 1.3695 & 47.0943 & 1.3690 & 45.6702 & 1.3697 & 42.8128 \\
\hline 1.9290 & 41.8841 & 1.9290 & 43.2395 & 1.9291 & 41.3387 & 1.9301 & 37.1289 \\
\hline 2.3443 & 39.6925 & 2.3440 & 40.7104 & 2.3441 & 38.6433 & 2.3449 & 35.5210 \\
\hline 2.6737 & 38.9383 & 2.6737 & 40.3341 & 2.6736 & 37.2610 & 2.6739 & 34.2404 \\
\hline 2.9603 & 38.7273 & 2.9606 & 40.0501 & 2.9606 & 37.2329 & 2.9609 & 34.0800 \\
\hline 3.2145 & 37.9623 & 3.2145 & 39.5702 & 3.2150 & 36.6042 & 3.2153 & 33.5487 \\
\hline 3.4387 & 37.7055 & 3.4384 & 38.8742 & 3.4389 & 36.0507 & 3.4392 & 33.3437 \\
\hline 3.6431 & 37.3487 & 3.6427 & 38.5567 & 3.6434 & 35.7337 & 3.6438 & 33.2001 \\
\hline 3.8300 & 37.2325 & 3.8297 & 38.4766 & 3.8302 & 35.4004 & 3.8305 & 32.9063 \\
\hline 4.0021 & 37.0003 & 4.0018 & 38.2943 & 4.0024 & 35.0418 & 4.0028 & 32.6246 \\
\hline 4.1638 & 36.7738 & 4.1636 & 38.0215 & 4.1641 & 34.3919 & 4.1648 & 32.5367 \\
\hline 4.3092 & 36.7552 & 4.3089 & 37.9706 & 4.3096 & 33.9399 & 4.3101 & 32.4657 \\
\hline 4.4470 & 36.7089 & 4.4468 & 37.8627 & 4.4473 & 33.7157 & 4.4476 & 32.4456 \\
\hline 4.5784 & 36.7041 & 4.5781 & 37.5717 & 4.5787 & 33.4294 & 4.5793 & 32.3608 \\
\hline 4.7013 & 36.6378 & 4.7011 & 37.4503 & 4.7017 & 33.3104 & 4.7021 & 32.3157 \\
\hline
\end{tabular}

شكل (1-7): العلاقة بين الجذر التربيعي لتراكيز معقدات حامض الأرجنين مع المنغيز إزاء المواصلة المكافئة بنسب مختلفة في محلول السكروز بارجة (310.16 مطلقة)

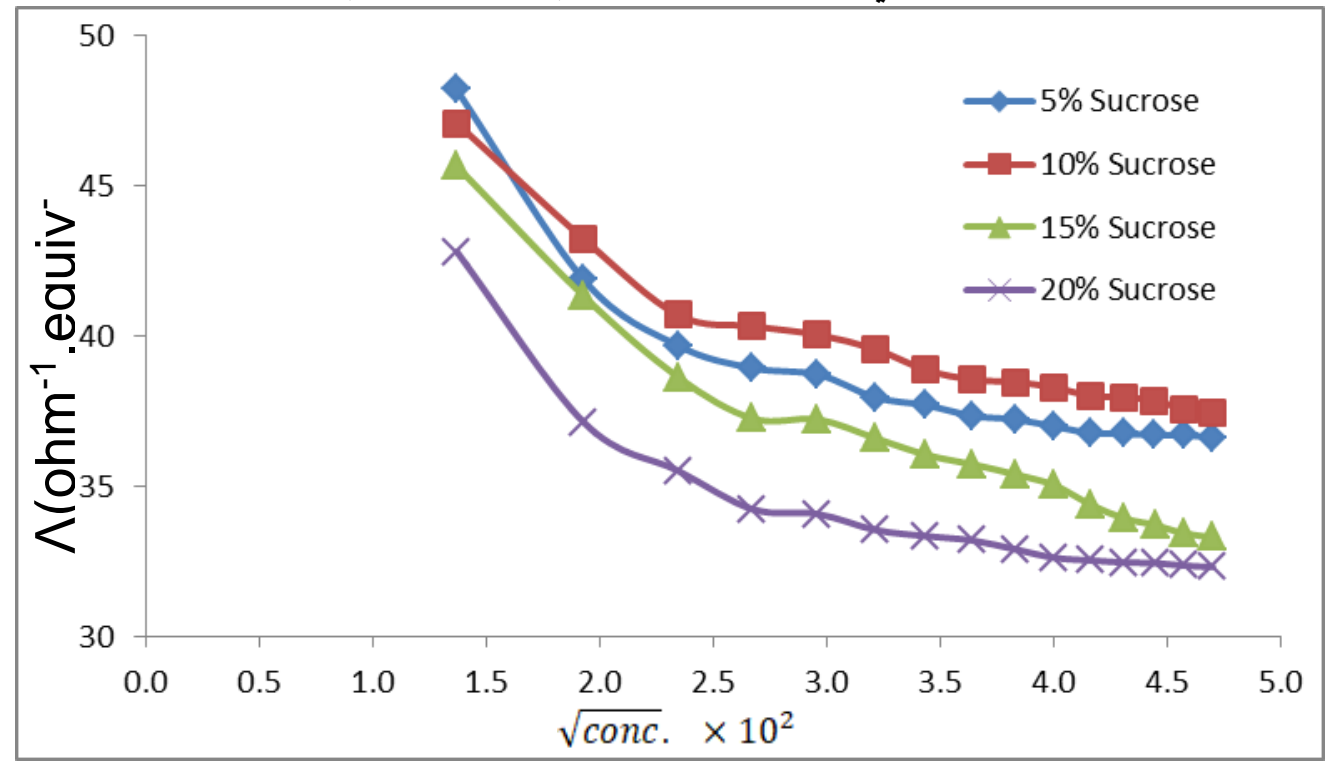


جدول (10-10): يوضح قيم الجذر التربيعي لتراكيز مختلفة لمعقدات الحامض الأميني الأرجنين مع النيكل إزاء المواصلة المكافئة بنسب مختلفة من محلول الكلوكوز عند درجة حرارة (310.16 مطلقة)

\begin{tabular}{|c|c|c|c|c|c|c|c|}
\hline \multicolumn{2}{|c|}{$5 \%$ Glucose } & \multicolumn{2}{c|}{$10 \%$ Glucose } & \multicolumn{2}{c|}{$15 \%$ Glucose } & \multicolumn{2}{c|}{$20 \%$ Glucose } \\
\hline$\sqrt{\text { conc. } \times 10^{2}}$ & $\Lambda$ equiv. & $N_{\text {conc. } \times 10^{2}}$ & $\Lambda$ equiv. & $\sqrt{\text { conc. } \times 10^{2}}$ & $\Lambda$ equiv. & $N_{\text {conc. } \times 10^{2}}$ & $\Delta$ equiv. \\
\hline 1.4307 & 55.2019 & 1.4314 & 52.7814 & 1.4310 & 51.0468 & 1.4240 & 49.3439 \\
\hline 2.0027 & 42.8121 & 2.0027 & 39.4274 & 2.0031 & 38.5215 & 1.9978 & 37.5989 \\
\hline 2.4293 & 37.3089 & 2.4296 & 34.1596 & 2.4298 & 32.6190 & 2.4258 & 32.8084 \\
\hline 2.7752 & 35.2733 & 2.7752 & 32.9874 & 2.7759 & 31.6167 & 2.7720 & 30.6553 \\
\hline 3.0694 & 34.4093 & 3.0697 & 32.8729 & 3.0701 & 31.3456 & 3.0638 & 29.4831 \\
\hline 3.3254 & 34.0627 & 3.3259 & 32.8682 & 3.3261 & 31.2577 & 3.3204 & 29.2488 \\
\hline 3.5564 & 33.5368 & 3.5567 & 32.7920 & 3.5571 & 31.1966 & 3.5517 & 28.5831 \\
\hline 3.7646 & 32.3282 & 3.7645 & 32.7874 & 3.7654 & 31.1503 & 3.7583 & 28.4296 \\
\hline 3.9559 & 31.7381 & 3.9556 & 32.7823 & 3.9567 & 31.1023 & 3.9496 & 27.3764 \\
\hline 4.1276 & 31.5936 & 4.1277 & 32.6037 & 4.1284 & 30.5732 & 4.1235 & 26.9989 \\
\hline 4.2882 & 31.4411 & 4.2884 & 31.8306 & 4.2889 & 30.3155 & 4.2841 & 26.5111 \\
\hline 4.4352 & 31.4372 & 4.4355 & 31.6412 & 4.4360 & 29.6992 & 4.4312 & 26.2307 \\
\hline 4.5758 & 31.3628 & 4.5763 & 31.3062 & 4.5767 & 29.4204 & 4.5706 & 26.1630 \\
\hline 4.7058 & 31.3606 & 4.7064 & 30.5914 & 4.7066 & 29.1854 & 4.7009 & 26.0684 \\
\hline 4.8288 & 31.2735 & 4.8292 & 30.3531 & 4.8296 & 29.1656 & 4.8239 & 26.0667 \\
\hline
\end{tabular}

شكل (1-8): العلاقة بين الجذر التربيعي لتراكيز معقدات حامض الأرجنين مع النيكل إزاء المواصلة المكافئة بنسب مختلفة في محلول الكلوكوز بارجة (310.16 مطلقة)

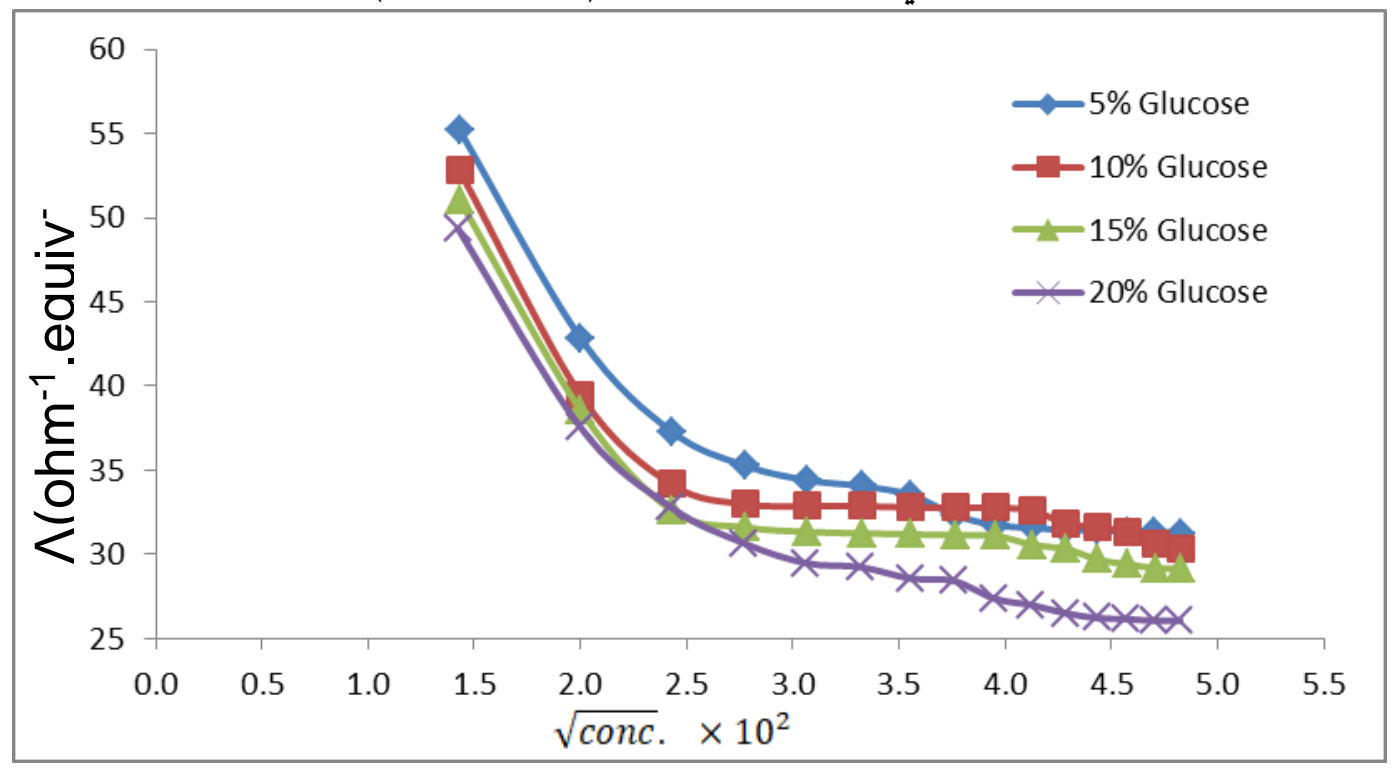


جدول (1-11): يوضح قيم الجذر التربيعي لتراكيز مختلفة لمعقدات الحامض الأميني الأرجنين مع النيكل إزاء المواصلة المكافئة بنسب مختلفة من محلول الفركتوز عند درجة حرارة (310.16 مطلقة)

\begin{tabular}{|c|c|c|c|c|c|c|c|}
\hline \multicolumn{2}{|c|}{$5 \%$ Fructose } & \multicolumn{2}{|c|}{$10 \%$ Fructose } & \multicolumn{2}{|c|}{$15 \%$ Fructose } & \multicolumn{2}{|c|}{$20 \%$ Fructose } \\
\hline$\sqrt{\text { conc. } \times 10^{2}}$ & $\Lambda$ equiv. & $\sqrt{\text { conc. } \times 10^{2}}$ & $\Delta$ equiv. & $\sqrt{\text { conc. } \times 10}$ & $\Delta$ equiv. & $\sqrt{\text { conc. } \times 10^{2}}$ & $\Delta$ equiv. \\
\hline 1.3958 & 65.9832 & 1.3958 & 62.5367 & 1.3889 & 56.0140 & 1.3951 & 51.4399 \\
\hline 1.9548 & 50.9768 & 1.9548 & 43.5058 & 1.9496 & 41.4508 & 1.9552 & 38.4543 \\
\hline 2.3678 & 38.6567 & 2.3679 & 34.7991 & 2.3637 & 36.3442 & 2.3689 & 34.0387 \\
\hline 2.7028 & 36.7953 & 2.7028 & 30.2432 & 2.6999 & 33.8918 & 2.7043 & 31.2467 \\
\hline 2.9890 & 36.4454 & 2.9890 & 28.8841 & 2.9871 & 32.1592 & 2.9906 & 28.6873 \\
\hline 3.2415 & 35.5207 & 3.2415 & 28.7096 & 3.2398 & 32.0038 & 3.2427 & 27.7738 \\
\hline 3.4664 & 35.4295 & 3.4664 & 28.5573 & 3.4656 & 31.0615 & 3.4677 & 27.6284 \\
\hline 3.6680 & 34.7641 & 3.6680 & 28.4871 & 3.6675 & 30.5243 & 3.6696 & 27.6267 \\
\hline 3.8529 & 34.2021 & 3.8529 & 28.3308 & 3.8526 & 30.1877 & 3.8542 & 27.2069 \\
\hline 4.0228 & 33.7532 & 4.0228 & 28.2289 & 4.0222 & 29.5810 & 4.0242 & 26.5415 \\
\hline 4.1794 & 33.6151 & 4.1795 & 28.1219 & 4.1790 & 29.3492 & 4.1806 & 26.1788 \\
\hline 4.3273 & 33.5034 & 4.3274 & 27.8823 & 4.3272 & 29.3241 & 4.3285 & 26.0624 \\
\hline 4.4639 & 33.4690 & 4.4640 & 27.6805 & 4.4640 & 29.1896 & 4.4650 & 26.0336 \\
\hline 4.5920 & 33.4682 & 4.5920 & 27.5342 & 4.5922 & 29.1890 & 4.5929 & 25.8615 \\
\hline 4.7116 & 33.4088 & 4.7117 & 27.5278 & 4.7119 & 29.1339 & 4.7126 & 25.8085 \\
\hline
\end{tabular}

شكل (1-9): العلاقة بين الجذر التربيعي لتراكيز معقدات حامض الأرجنين مع النيكل إزاء المواصلة المكافئة بنسب

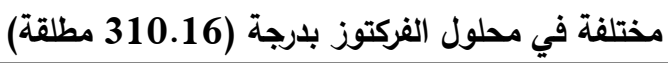

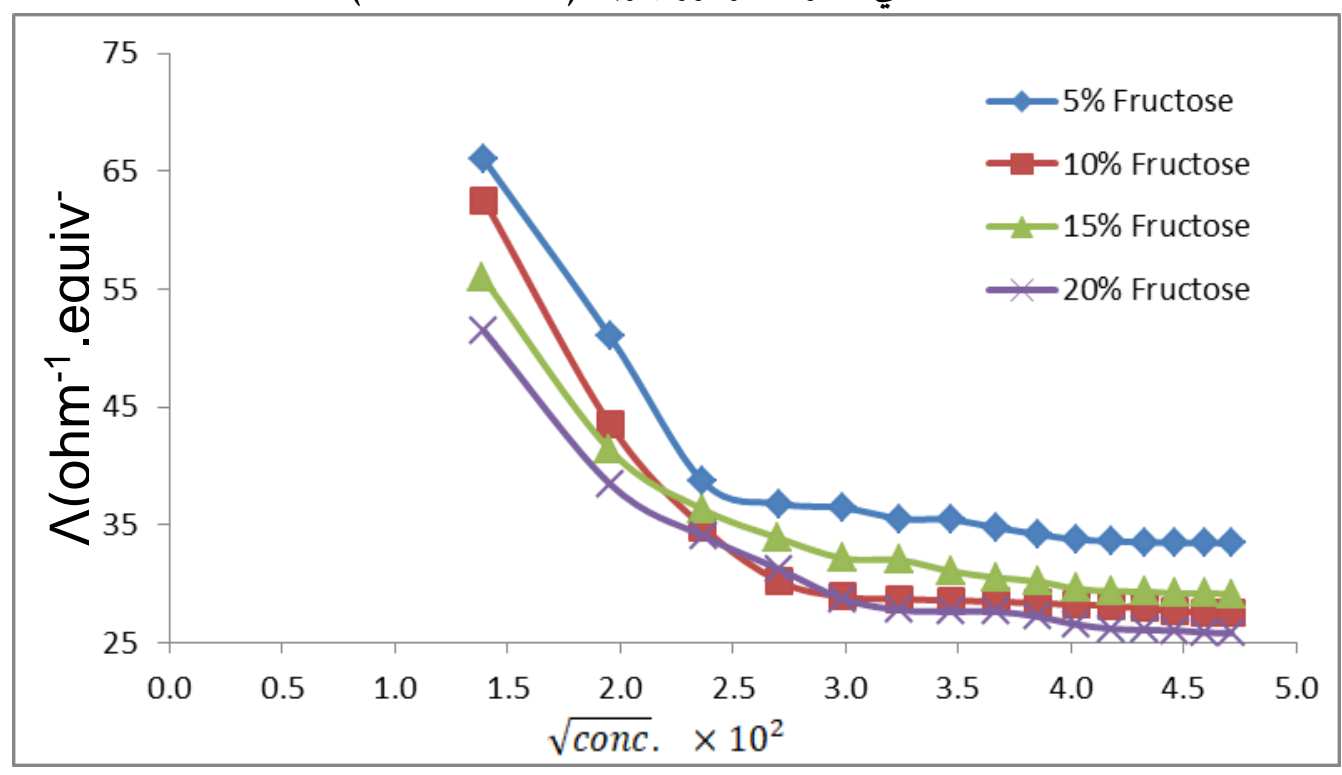

جدول (1-12): يوضح قيم الجذر التربيعي لتراكيز مختلفة لمققدات الحامض الأميني الأرجنين مع النيكل إزاء المواصلة المكافئة بنسب مختلفة من محلول السكروز عند درجة حرارة (310.16 مطلقة) 
قياسات التوصيلية الكهربائية لظاهرة التجمع الايوني لمعقدات الحامض الاميني الارجنين مع عدد من العناصر ...

\begin{tabular}{|c|c|c|c|c|c|c|c|}
\hline \multicolumn{2}{|c|}{$5 \%$ Sucrose } & \multicolumn{2}{|c|}{$10 \%$ Sucrose } & \multicolumn{2}{|c|}{$15 \%$ Sucrose } & \multicolumn{2}{|c|}{$20 \%$ Sucrose } \\
\hline$\sqrt{\text { conc. } \times 10}$ & $\Lambda$ equiv. & $\sqrt{\text { conc. } \times 10^{2}}$ & $\Delta$ equiv. & $\sqrt{\text { conc. } \times 10}$ & $\Delta$ equiv. & $\sqrt{\text { conc. } \times 10^{2}}$ & $\Delta$ equiv. \\
\hline 1.4307 & 48.7563 & 1.4307 & 44.9516 & 1.4301 & 41.9251 & 1.4302 & 40.3508 \\
\hline 2.0027 & 40.8404 & 2.0027 & 36.5955 & 2.0017 & 37.4982 & 2.0016 & 37.7404 \\
\hline 2.4293 & 37.0776 & 2.4291 & 34.1715 & 2.4272 & 34.4171 & 2.4272 & 35.6299 \\
\hline 2.7752 & 35.2197 & 2.7751 & 33.7002 & 2.7728 & 33.9446 & 2.7728 & 34.5780 \\
\hline 3.0694 & 35.0960 & 3.0694 & 33.2782 & 3.0669 & 33.7344 & 3.0669 & 34.1685 \\
\hline 3.3254 & 34.3914 & 3.3253 & 33.1796 & 3.3225 & 33.5261 & 3.3226 & 33.7634 \\
\hline 3.5564 & 33.8242 & 3.5563 & 32.5507 & 3.5527 & 32.8030 & 3.5528 & 33.3241 \\
\hline 3.7646 & 32.9728 & 3.7646 & 32.4553 & 3.7602 & 32.7110 & 3.7603 & 33.0558 \\
\hline 3.9559 & 32.2427 & 3.9557 & 32.3291 & 3.9502 & 32.6793 & 3.9503 & 32.9813 \\
\hline 4.1276 & 32.1837 & 4.1275 & 32.1746 & 4.1217 & 32.5950 & 4.1218 & 32.8866 \\
\hline 4.2882 & 32.1534 & 4.2881 & 31.9719 & 4.2819 & 32.2508 & 4.2819 & 32.5679 \\
\hline 4.4352 & 32.1206 & 4.4351 & 31.8078 & 4.4282 & 32.0918 & 4.4283 & 32.4418 \\
\hline 4.5758 & 32.0856 & 4.5758 & 31.6488 & 4.5686 & 31.9485 & 4.5686 & 32.3422 \\
\hline 4.7058 & 32.0429 & 4.7058 & 31.4483 & 4.6981 & 31.8156 & 4.6982 & 32.3413 \\
\hline 4.8288 & 32.0023 & 4.8288 & 31.1801 & 4.8205 & 31.5019 & 4.8205 & 31.9506 \\
\hline
\end{tabular}

شكل (1-10): العلاقة بين الجذر التربيعي لتراكيز معقدات حامض الأرجنين مع النيكل إزاء المواصلة المكافئة بنسب مختلفة في محلول السكروز بدرجة (310.16 مطلقة)

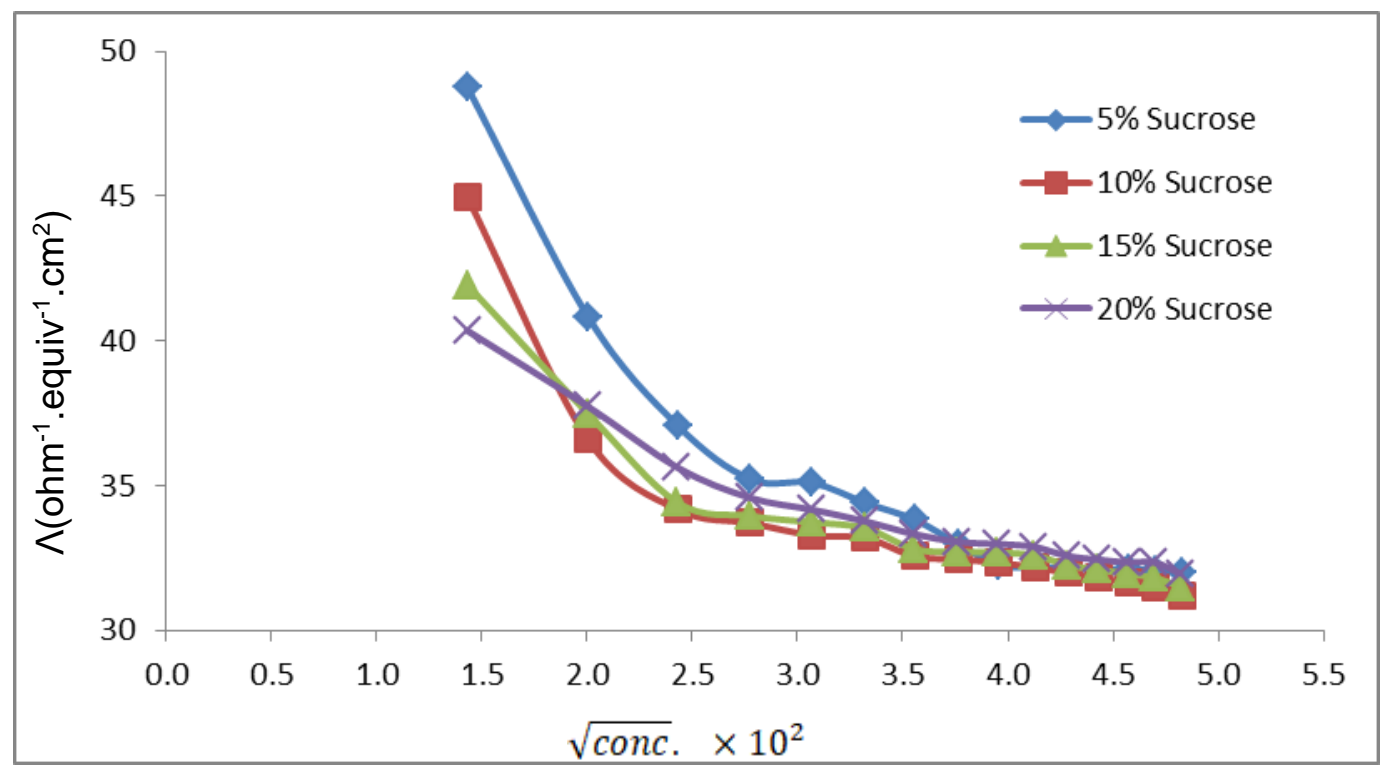

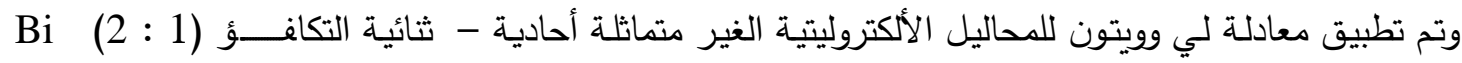
single - univalent electrolytes :310.16 مطلقة) بصيغتها الموضحة أدناه:

$\lambda_{\mathrm{j}}=\lambda_{\mathrm{j}}^{\circ}\left\{1+\mathrm{Z} \sum_{\mathrm{p}=2}^{\mathrm{s}} \mathrm{X}_{\mathrm{j}}^{\mathrm{p}} \sum_{\mathrm{p}=1} \mathrm{tvX}_{\mathrm{v}}^{\mathrm{p}}\left[\mathrm{A}_{\mathrm{v}}^{\mathrm{p}}(\mathrm{t})(\beta \mathrm{K})+\mathrm{B}_{\mathrm{v}}^{\mathrm{p}}(\mathrm{t})(\beta \mathrm{K})^{2}+\mathrm{C}_{\mathrm{v}}^{\mathrm{p}}(\mathrm{t})(\beta \mathrm{K})^{3}\right]\right\}$

$-\frac{\left|\mathrm{Z}_{\mathrm{j}}\right|(\mathrm{KT})}{2(1+\mathrm{t})}\left\{1+\mathrm{V}_{\mathrm{j}}^{(1)}(\mathrm{t})(\beta \mathrm{K})+\mathrm{V}_{\mathrm{j}}^{2}(\mathrm{t})(\beta \mathrm{K})^{2}+\pi_{\mathrm{j}}^{(5)} \mathrm{t} / 6\right\}$

إذ إن المعادلات

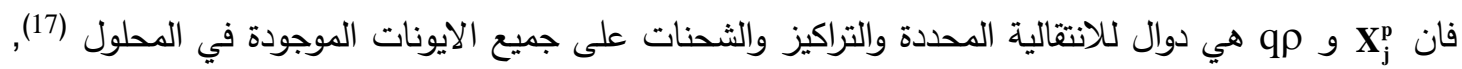


وتم تحليل البيانات التي تتضمن قيم كل من التراكيز والمواصلة المكافئة باستعمال البرنامج AM1 بعد إعطائه

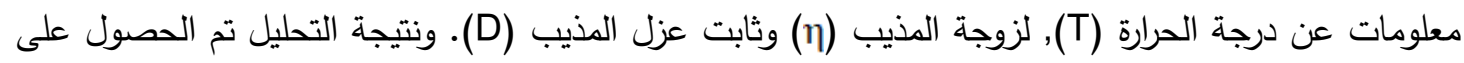

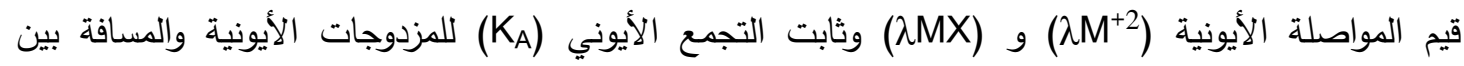

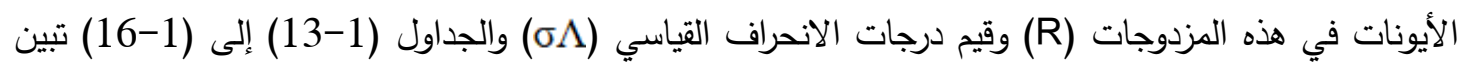

هذه النتائج:

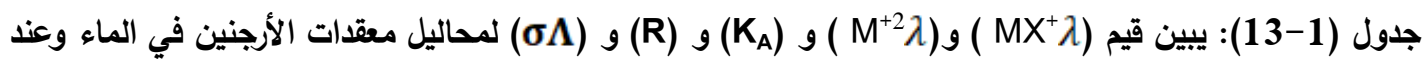

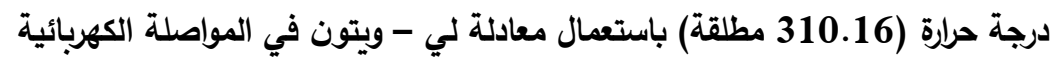

\begin{tabular}{|c|c|c|c|c|c|}
\hline الصيغة الوضعية للمعقد & $\mathrm{K}_{\mathrm{A}}$ & $\lambda \mathrm{M}^{+2}$ & $\lambda \mathrm{MX}^{+}$ & $\mathrm{R}$ & $\sigma \Lambda$ \\
\hline$\left[\mathrm{Fe}\left(\mathrm{C}_{6} \mathrm{H}_{14} \mathrm{~N}_{4} \mathrm{O}_{2}\right)_{3}\right] \mathrm{Cl}_{2}$ & 1135 & 35 & 1.0 & 4.5 & 0.0638 \\
\hline$\left[\mathrm{Mn}\left(\mathrm{C}_{6} \mathrm{H}_{14} \mathrm{~N}_{4} \mathrm{O}_{2}\right)_{3}\right] \mathrm{Cl}_{2}$ & 1090 & 38 & 1.0 & 5.6 & 0.0771 \\
\hline$\left[\mathrm{Ni}\left(\mathrm{C}_{6} \mathrm{H}_{14} \mathrm{~N}_{4} \mathrm{O}_{2}\right)_{3}\right] \mathrm{Cl}_{2}$ & 1300 & 30 & 1.0 & 7.0 & 0.0812 \\
\hline
\end{tabular}

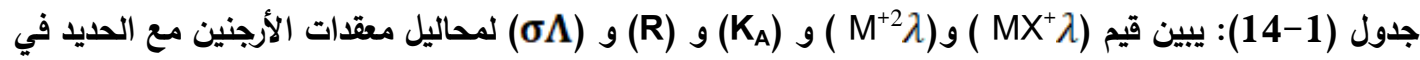

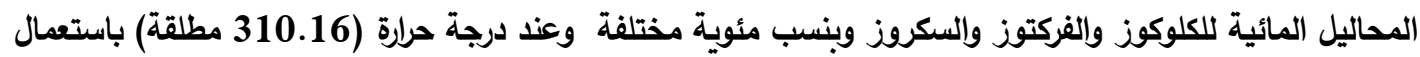
معادلة لي - ويتون في المواصلة الكهربائية

\begin{tabular}{|c|c|c|c|c|c|c|c|c|c|c|c|c|c|c|c|}
\hline \multirow{2}{*}{ W\% } & \multicolumn{5}{|c|}{ Glucose } & \multicolumn{5}{|c|}{ Fructose } & \multicolumn{5}{|c|}{ Sucrose } \\
\hline & $\mathrm{K}_{\mathrm{A}}$ & $\lambda \mathrm{M}^{+2}$ & $\lambda \mathrm{MX}^{+}$ & $\mathrm{R}$ & $\sigma \Lambda$ & $\mathrm{K}_{\mathrm{A}}$ & $\lambda \mathrm{M}^{+2}$ & $\lambda \mathrm{MX}^{+}$ & $\mathrm{R}$ & $\sigma \Lambda$ & $\mathrm{K}_{\mathrm{A}}$ & $\lambda \mathrm{M}^{+2}$ & $\lambda \mathrm{MX}^{+}$ & $\mathrm{R}$ & $\sigma \Lambda$ \\
\hline $5 \%$ & 1150 & 27 & 1.0 & 8.0 & & 1585 & 30 & & 9.0 & & & 2 & & 8.8 & \\
\hline $10^{c}$ & & 2 & & 9.0 & & & 29 & & & & & 19 & & 8.5 & \\
\hline 15 & 46 & 24 & & 7.0 & & 795 & 28 & & 9.3 & & 205 & 18 & & 8.2 & 03 \\
\hline $20 \%$ & 1350 & 23.5 & 1.0 & 8.0 & 0.07 & 1830 & 27 & 1.0 & 7.8 & 0.08 & 1890 & 16.9 & 1.0 & 8.9 & 0.03 \\
\hline
\end{tabular}

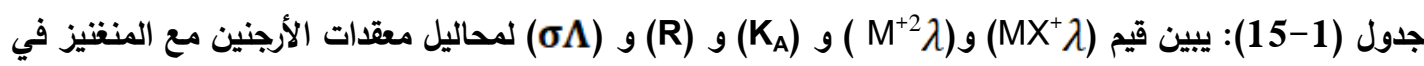

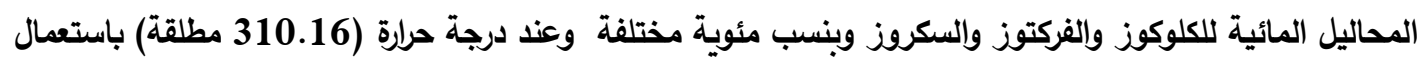
معادلة لي - ويتون في المواصلة الكهربائية

\begin{tabular}{|c|c|c|c|c|c|c|c|c|c|c|c|c|c|c|c|}
\hline \multirow{2}{*}{ W\% } & \multicolumn{5}{|c|}{ Glucose } & \multicolumn{5}{|c|}{ Fructose } & \multicolumn{5}{|c|}{ Sucrose } \\
\hline & $\mathrm{K}_{\mathrm{A}}$ & $\lambda \mathrm{M}^{+2}$ & $\lambda \mathrm{MX}^{+}$ & $\mathrm{R}$ & $\sigma \Lambda$ & $\mathrm{K}_{\mathrm{A}}$ & $\lambda \mathrm{M}^{-}$ & $\lambda \mathrm{MX}^{+}$ & $\mathrm{R}$ & $\sigma \Lambda$ & $\mathrm{K}_{\mathrm{A}}$ & $\lambda \mathrm{M}^{+}$ & $\lambda \mathrm{MX}^{+}$ & $\mathrm{R}$ & $\sigma \Lambda$ \\
\hline $5 \%$ & 1200 & 25 & 1.0 & 8.2 & 0.04 & 1325 & 28 & 1.0 & 8.7 & 0.04 & 1358 & 22 & 1.0 & 9.1 & 0.03 \\
\hline $10 \%$ & 1290 & 23 & 1.0 & 8.7 & 0.03 & 1337 & 27 & 1.0 & 9.1 & 0.03 & 1375 & 19.5 & 1.0 & 9.4 & .04 \\
\hline $15 \%$ & 1352 & 22 & 1.0 & 9.1 & 0.02 & 1365 & 25 & 1.0 & 9.5 & 0.04 & 1391 & 19 & 1.0 & 9.6 & 0.05 \\
\hline $20 \%$ & 1360 & 21.5 & 1.0 & 9.3 & 0.05 & 1380 & 23 & 1.0 & 9.8 & 0.04 & 330 & 18.1 & 1.0 & 9.8 & 0.03 \\
\hline
\end{tabular}

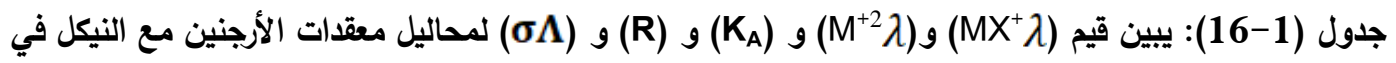

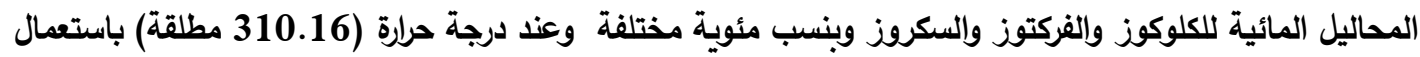
معادلة لي - ويتون في المواصلة الكهربائية

\begin{tabular}{|r|c|c|c|c|c|c|c|c|c|c|c|c|c|c|c|}
\hline \multirow{2}{*}{$\mathrm{W} \%$} & \multicolumn{9}{|c|}{ Glucose } & \multicolumn{4}{c|}{ Fructose } & \multicolumn{4}{c|}{ Sucrose } \\
\cline { 2 - 16 } & $\mathrm{K}_{\mathrm{A}}$ & $\lambda \mathrm{M}^{+2}$ & $\lambda \mathrm{MX}^{+}$ & $\mathrm{R}$ & $\sigma \Lambda$ & $\mathrm{K}_{\mathrm{A}}$ & $\lambda \mathrm{M}^{+2}$ & $\lambda \mathrm{MX}^{+}$ & $\mathrm{R}$ & $\sigma \Lambda$ & $\mathrm{K}_{\mathrm{A}}$ & $\lambda \mathrm{M}^{+2}$ & $\lambda \mathrm{MX}^{+}$ & $\mathrm{R}$ & $\sigma \Lambda$ \\
\hline $5 \%$ & 1357 & 13.8 & 1.0 & 8.4 & 0.04 & 1380 & 16.8 & 1.0 & 7.8 & 0.04 & 1384 & 10.7 & 1.0 & 7.9 & 0.04 \\
\hline $10 \%$ & 1365 & 11.3 & 1.0 & 8.9 & 0.05 & 1385 & 15.1 & 1.0 & 7.9 & 0.05 & 1390 & 9.0 & 1.0 & 8.7 & 0.06 \\
\hline $15 \%$ & 1370 & 10.0 & 1.0 & 8.9 & 0.05 & 1392 & 13.3 & 1.0 & 8.7 & 0.04 & 1395 & 8.8 & 1.0 & 8.8 & 0.04 \\
\hline $20 \%$ & 1379 & 9.5 & 1.0 & 9.5 & 0.07 & 1399 & 12.0 & 1.0 & 8.9 & 0.04 & 1409 & 7.3 & 1.0 & 8.9 & 0.05 \\
\hline
\end{tabular}

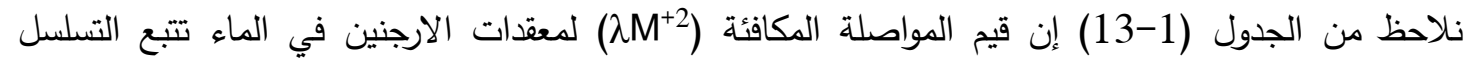


$\lambda \mathrm{M}^{+2}$ : Arginine Complexes of $\mathrm{Mn}^{+2}>$ Arginine Complexes of $\mathrm{Fe}^{+2}>$ Arginine Complexes of $\mathrm{Ni}^{+2}$

ويعود السبب لاختلاف انصاف الاقطار الايونية (الذرية) للعناصر الانتقالية الداخلة في تكوين المعقدات إذ يكون نصف القطر الايوني للمنغنيز أكبر من نصف القطر الايوني للحديد ,والحديد أكبر من النيكل وهذا ما أثبتته قيم

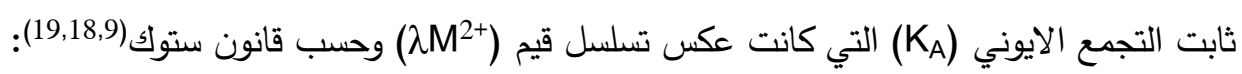
$\lambda \mathrm{i}^{\circ}=8.2 \times 10^{-2} \mathrm{Zi} / \eta \mathrm{ri}$

$$
\begin{aligned}
& \text { إذ أن:- }
\end{aligned}
$$

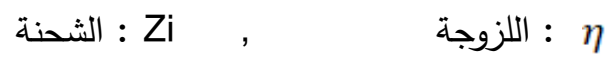

وتفسر قيم ثابت التجمع الايوني (KA) ان تجمع جزيئات المذيب في معقد الأرجنين مع المنغنيز أقل مما في

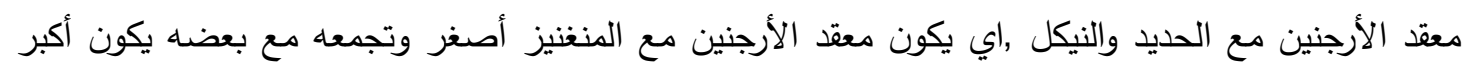

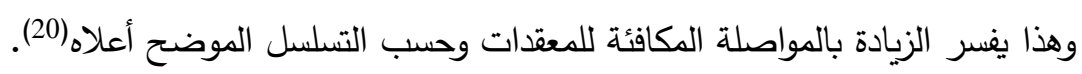
أما قيم (

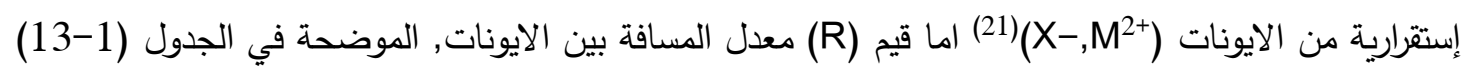
هي اعلى من قيمة ثابت جيرم (3.57A) وهذا يدل على ان التجمعات الايونية هي من نوع التجمعات المفصولة بزيئات المذيب Solvent separate ion pair (SSIP). وتعتمد قيمها على مدى التفاعلات التبادلية بين الايون والمذيب (ion_solvent) في المحلول(23,22).

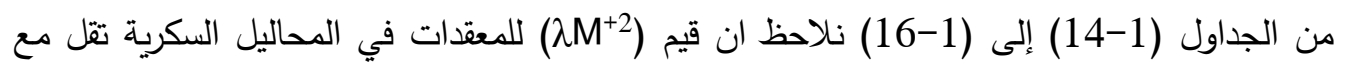

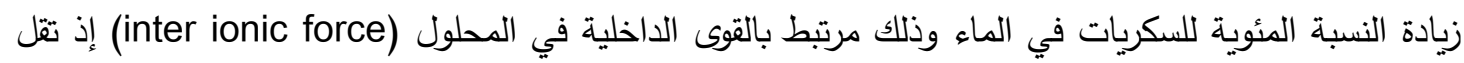
التداخلات الجزيئية من نوع أيون _أيون (ion_ion interactions) مع زيادة التداخلات الجزيئية من نوع (أيون

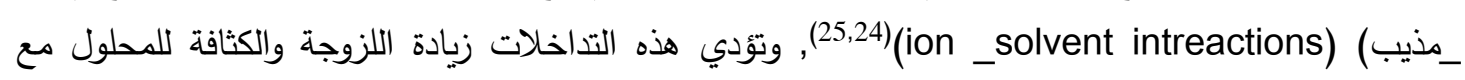
زيادة نسبة السكر بالماء مما يقلل من الانتقالية الايونية وحسب قانون ستوك(19), لذلك يكون ترتيب قيم المواصلة

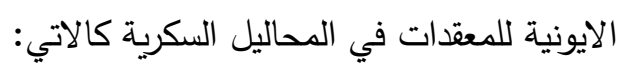

$\lambda \mathrm{M}^{2+}$ : Arginine Complexes $_{\text {(in aqueous fructose solutions ) }}>$ Arginine Complexes $_{\text {(in aqueous glucose }}$

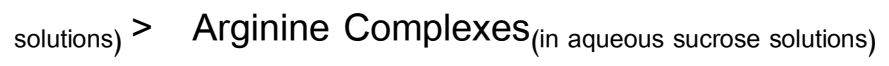
اما قيم ثابت التجمع الايوني (R) و (R) فإنها تزداد مع زيادة النسبة المئوية للسكر بالماء وهذا ما أكدته

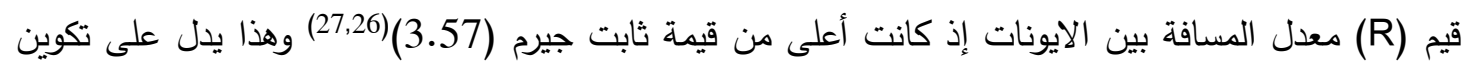
مزدوجات ايونية من نوع (SSIP) ويكون تسلسل قيم (KA) للمعقدات في المحاليل السكرية كالاتي:

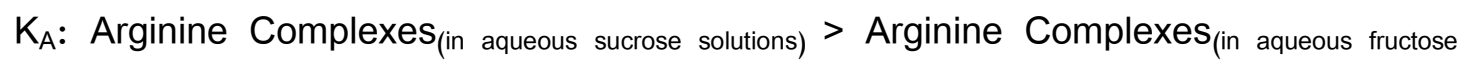
solutions) > Arginine Complexes (in aqueous glucose solutions)

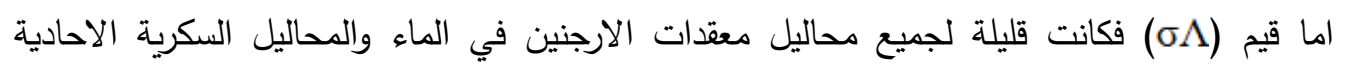
والثنائية المذكورة انفا ,مما يؤكد ان تطبيق معادلة لي _ويتون مناسب لهذه الدراسة ـ وتم حساب ناتج والدان لمعقدات الأرجنين الذي يوضح عملية التذاوب التي تتأثر بتغيير النسب المئوية للمحاليل السكرية, والجداول أدناه

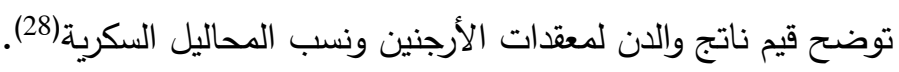
جدول (1-17): يبين قيم ناتج والدن لمعقد الأرجنين مع الحديد ونسب السكريات في الماء لاء عند درجة حرارة (310.16 


\begin{tabular}{|c|c|c|c|}
\hline Wt.\% & Glucose & Fructose & Sucrose \\
\hline $5 \%$ & 19.1268 & 20.8200 & 14.5780 \\
\hline $10 \%$ & 18.5725 & 21.2599 & 14.6984 \\
\hline $15 \%$ & 18.4176 & 21.0532 & 14.9238 \\
\hline $20 \%$ & 18.8446 & 21.1842 & 14.2483 \\
\hline
\end{tabular}

شكل (1-11): ناتج واللن لمعقد الحديد مع الأرجنين مقابل النسبة المئوية للسكريات في الماء

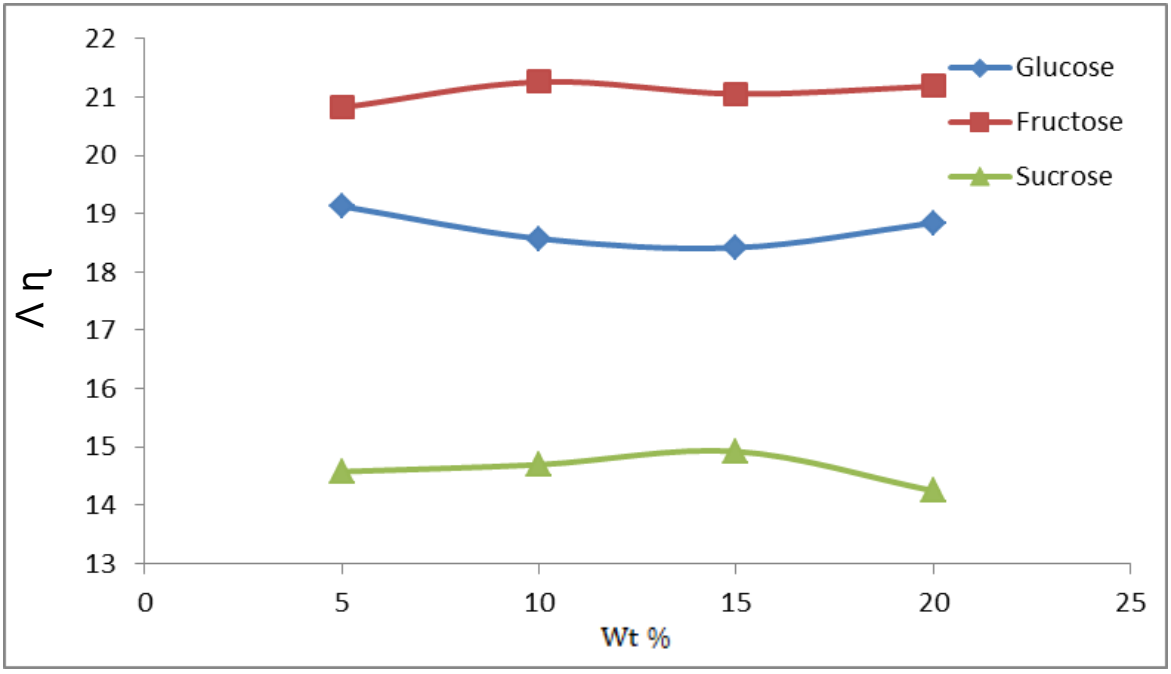

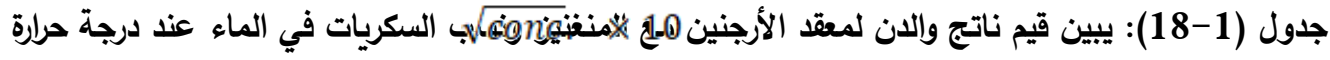

(310.16) مطلة)

\begin{tabular}{|c|c|c|c|}
\hline Wt.\% & Glucose & Fructose & Sucrose \\
\hline $5 \%$ & 17.7100 & 19.4320 & 16.0358 \\
\hline $10 \%$ & 17.0867 & 19.7937 & 15.0852 \\
\hline $15 \%$ & 16.8828 & 18.7975 & 15.7529 \\
\hline $20 \%$ & 17.2408 & 18.0458 & 15.2601 \\
\hline
\end{tabular}

شكل (1-12): ناتج واللان لمعقد المنفتيز مع الأرجنين مقابل النسبة المئوية للسكريات في الماء

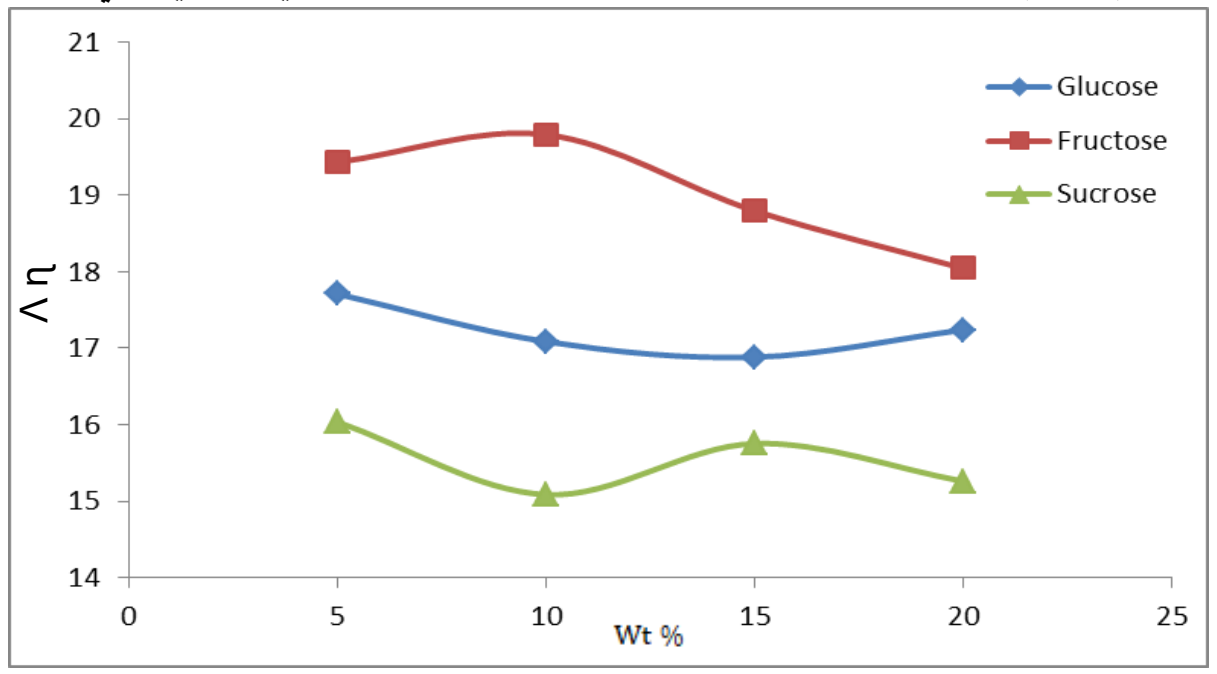

جدول (1-19): يبين قيم ناتج والدن لمعقد الأرجنين مع النيكل ونسب السكريات في الماء عند درجة حرارة (310.16

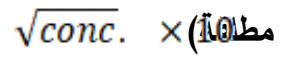


قياسات التوصيلية الكهبائية لظاهرة التجمع الايوني لمعقدات الحامض الاميني الارجنين مع عدد من العناصر ...

\begin{tabular}{|c|c|c|c|}
\hline Wt.\% & Glucose & Fructose & Sucrose \\
\hline $5 \%$ & 9.7759 & 11.6592 & 7.7992 \\
\hline $10 \%$ & 8.3947 & 11.0698 & 6.9624 \\
\hline $15 \%$ & 7.6740 & 10.0002 & 7.2960 \\
\hline $20 \%$ & 7.6180 & 9.4152 & 6.1546 \\
\hline
\end{tabular}

شكل (13-13): ناتج والدن لمعقد النيكل مع الأرجنين مقابل النسبة المئوية للسكريات في الماء

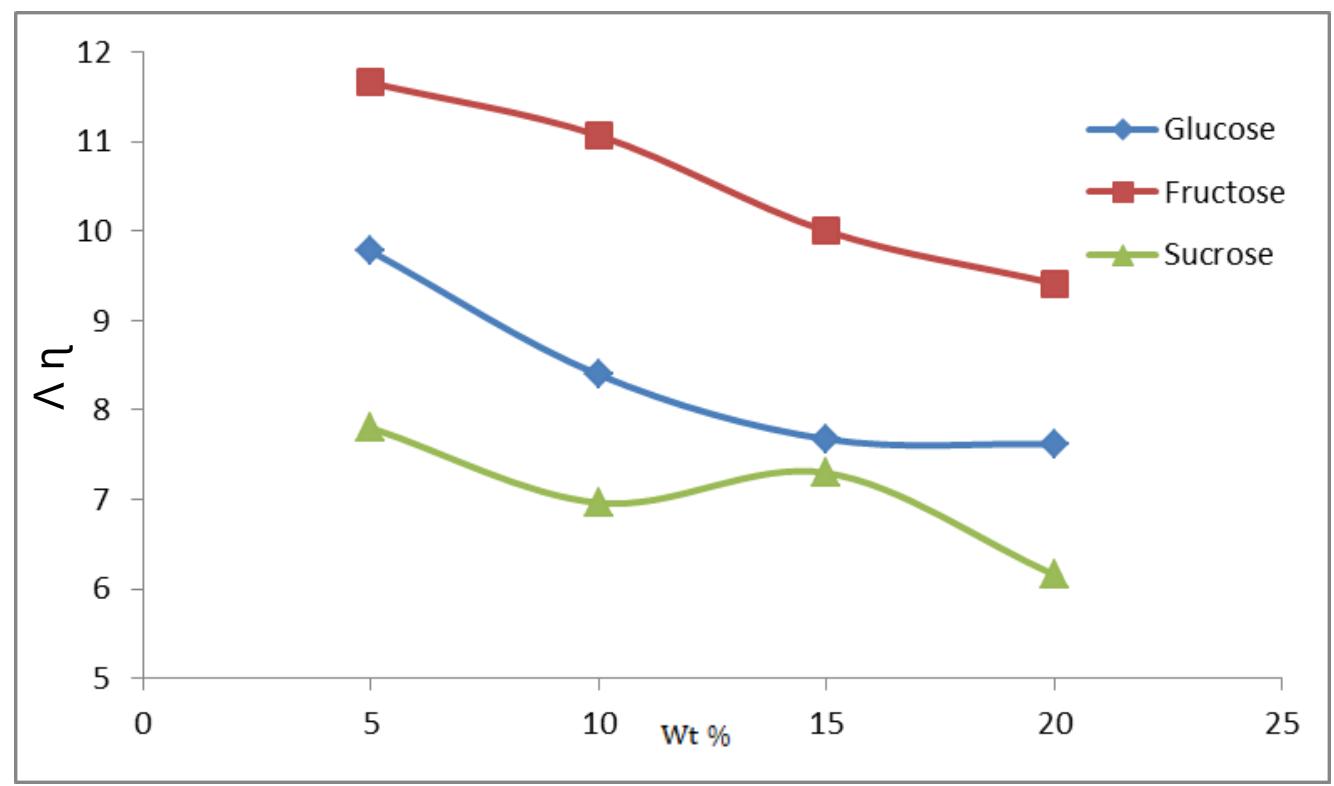

$\sqrt{\text { conc. }} \times 10$

المصادر

1) Grecu I., Sandulescu R. and Neamtu M., Rev.Chim., 37(7): 589_595 (1986).

2) El-Said A.I, Zidan A. S, El-Meligy M.S., Aly A. Aand Mohamened O.F., Synth. React.Inorg .Met-Org. Chem.31(4): 633-648 (2001).

3) Wilkinson S.G, Gillard R.D. and Mcleverty J.A., Regramon Press Oxford, 2 (1):740-742(1989).

4) Al-Allaf Y. O. and Abdulrahman H. A., Tik, j. Sci., No.16, Vol.1, PP 86-91, (2011).

5) Al-Allaf Y. O. and Abdulrahman H. A., Arab. J. Sci. Eng ISSN1319-8025, DOL10.1007/S13369-012-0259-5, (2012).

6) Al-Allaf Y. O., Al-Tamer M. Y. and Abdulfattah M. N., Raf.J.Sci., Vol.24, No.6, PP45-60, (2013).

7) Rabindar R.P, Radhika M. and Manjula P.,J.Chem.Sci.,117(3):239-246(2005).

8) Negoiu M., Rosu T., Saramet I. and Matei C.A., Chem. Anulx IV(II):129-133 (2005).

9) Al-Mashhadany M.N.A., MSc. Thesis, College of Science, University of Mosul (2009). (In Arabic).

10) Al-Saab F.M., MSc. Thesis. Thesis, College of Science, University of Mosul (2011). (In Arabic)

11) Hammed Y.O., T hanon F.A and Hani A.M, National Jornal of Chemisty, 25:111-123(2007).

12) Akrawi B.A, Jameil A.M, Abed A.G., Raf.J.Sci, 24(1):55-69 (2013). 
13) Ribeiro A.C.F., Ortona O., Simoes S.M.N., Santos C.I.A.V., Prazeres P.A., Valente A.JM., Lobo V.M.M and Burrows H.D, J.Chem.Eng.Data 51: 1836-1840 (2006).

14) Lee W.H., Wheaton R.J., J.Chem. Farady II (75):1128(1979).

15) Lerine I.N., "Physical Chemistry". $5^{\text {th }}$ Ed.MC. Graw-Hill companies, London (2002).

16) Palmer W.G., "Experimental physical Chemistry", Combridge, London(1954).

17) W.H.Lee and R.J.Wheaton, "Conductance of Symmetrical, Unsymmetrical and Mixed Electrolytes", J.CS Farady II, 75, 1128, (1979) .

18) Akrawi B.A., Ali A.M.J., National Jornal of Chemistry 31:491-500 (2008).

19) Al- Mustawi Y.O.H., Ph.D.Thesis, College of science, University of Mosul (2002), (In English).

20) Al-Allaf Y.O. and Abdul Rahman H.A., Tik-J.Sci.,17(1):233-237 (2012).

21) Hameed Y.O.,Shareef O.A. and Hani A.M., Tik-J.Sci.,11(2):324-327 (2006).

22) Lee W.H. and Wheaton R.J., J.Chem.Soc.Farady Trans. 74(2):743 (1978).

23) Lee W.H. and Wheaton R.J.,J.Chem.Soc.Farady Trans.75(2):1128 (1979).

24) Gregorowiz J., Bald A., Zejgis A.S. and Chmiele W.A., J.Mol.Liq., 84:149-160, (2000).

25) Gregorowiz J., Bald A., Zejgis A.S. and Zurada M.,J.Mol.Liq., 79:167-176 (1999).

26) Gomaa E.A., Al-Jahdalli B.M., American Journal of Cond. Matt.Phys., 2(1):1216 (2012).

27) Al-Sattar A.A., Raf.J.Sci.,13(3):9-18 (2002).

28) N. A. Hikmat, Irq. J. Chem., Vol. 28, No. 1, ( 2002). 